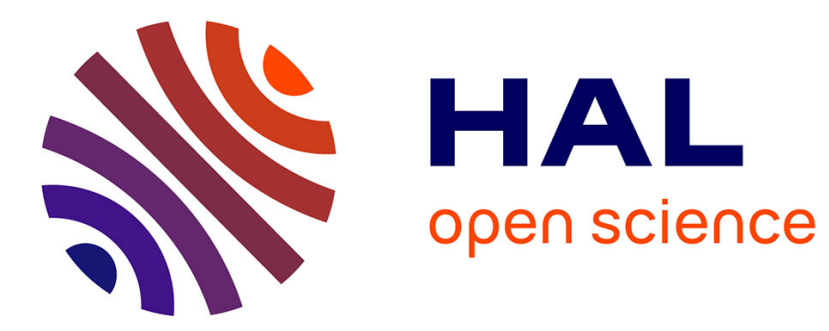

\title{
Mathematical analysis of a wave energy converter model
} Arnaud Rougirel

\section{To cite this version:}

Arnaud Rougirel. Mathematical analysis of a wave energy converter model. 2012. hal-00678055

\section{HAL Id: hal-00678055 \\ https://hal.science/hal-00678055}

Preprint submitted on 12 Mar 2012

HAL is a multi-disciplinary open access archive for the deposit and dissemination of scientific research documents, whether they are published or not. The documents may come from teaching and research institutions in France or abroad, or from public or private research centers.
L'archive ouverte pluridisciplinaire HAL, est destinée au dépôt et à la diffusion de documents scientifiques de niveau recherche, publiés ou non, émanant des établissements d'enseignement et de recherche français ou étrangers, des laboratoires publics ou privés. 


\title{
MATHEMATICAL ANALYSIS OF A WAVE ENERGY CONVERTER MODEL
}

\author{
ARNAUD ROUGIREL
}

\begin{abstract}
In a context where sustainable development should be a priority, Orazov et al. have proposed in 2010, an excitation scheme for buoy-type ocean wave energy converter. The simplest model for this scheme is a non autonomous piecewise linear second order differential equation. The goal of that paper is to give a mathematical framework for this model and to highlight some properties of its solutions. In particular, we will look at bounded and periodic solutions, and compare the energy performance of this novel WEC with respect to the one of wave energy converter without mass modulation.
\end{abstract}

\section{INTRODUCTION}

In the recent years, sustainable development has become a great challenge. Because of the crisis of fossil fuels, alternative sources of energy must be found. Moreover, decades of non sustainable development have produced pollution, climate disturbance and loss of biodiversity. Thus it is preferable to seek for novel sources of energy with low impact on the environment.

In this respect, ocean waves provides a important source of renewable energy. Mechanical devices that harvest energy stored in ocean waves are called wave energy converter (WEC): see [OOS10].

Basically, a WEC is a floating body with a power takeoff system. It uses the vertical oscillations induced by waves to produce electrical current. In order to optimize the harvesting capabilities of a WEC, Orazov et al. have proposed in 2010 a novel excitation method based on state-dependent mass modulations. We refer to [OOS10] for detailed explanations and to

http://me.berkeley.edu/ bayram/wec/water_intake_animation.html for an animation of this mechanism. Anyway, let us explain briefly how it works. The WEC can change its mass by holding back or ejecting water from its floats: this is mass modulation. The new idea of Orazov et al. is that the mass change occurs when the system is in a precise state : this is state-dependent mass modulation.

If the square of the vertical position starts to increase - i.e. $\frac{1}{2} \frac{\mathrm{d}}{\mathrm{d} t} x^{2}=x \dot{x}>0$ - then mass is added. When $x \dot{x}$ becomes negative, the water is ejected. This gives rises to quasilinear equation. Orazov et al. have proposed the following simple model

$$
\begin{array}{ll}
\ddot{x}+2 \omega \delta \dot{x}+\omega x=\omega g & \text { if } x \dot{x}>0 \\
\ddot{x}+2 \delta \dot{x}+x=g & \text { if } x \dot{x}<0 .
\end{array}
$$

Date: March 12, 2012. 
Here $x$ is the rescaled vertical position, $\dot{x}=\frac{\mathrm{d}}{\mathrm{d} t} x$ and $g: \mathbb{R} \rightarrow \mathbb{R}$ is a forcing term accounting for waves excitation. The positive constant $\delta$ is a damping coefficient and the parameter $\omega$ represents the mass added, namely

$$
\omega=\frac{M}{M+\Delta M}
$$

where $M$ is the mass of the WEC and $\Delta M$ the mass modulation.

If $\Delta M=0$ i.e. $\omega=1$ then there is no mass modulation and the model is linear. Otherwise, (1.1) is a piecewise linear system. The goal of that paper is to give a mathematical framework to equation (1.1) and to highlight some properties of its solutions. In particular, we will look at bounded and periodic solutions, and compare the energy performance of this novel WEC with respect to the one of WEC without mass modulation.

In the sequel, we will introduce our main results. As a matter of fact, solutions to (1.1) are $C^{1}$ functions thus the well posedness of (1.1) will be achieved with a continuous dynamical system approach. Remark that it should be possible to use alternatively the less standard theory of (discontinuous) piecewise linear system (see for instance [dBBCK08]) which models for example, bilinear or impact oscillators.

Regarding well posedness, the main point is to define the vector field on $x \dot{x}=0$. That is to say, at each point $M$ where $x \dot{x}=0$, we have to decide if the solution will enter in $x \dot{x}>0$ or either in $x \dot{x}<0$. In the former case, we set at the point $M$ the vector field defined by the first equation of (1.1). Since we are looking for oscillating solutions we will eliminate solutions that remain on $x \dot{x}=0$. However, since the system is non autonomous, it may happen that the trajectory leaves $x \dot{x}>0$ at time $t_{0}$ but enters again in $x \dot{x}>0$ for $t>t_{0}, t$ close to $t_{0}$. This occurs for instance if 0 is a local munimum of $x \dot{x}$. Under non degeneracy conditions on $g$, we are able to give, in Theorem 3.1, an existence and uniqueness result. The key point is that the flows defined by the two equations of (1.1) are consistent on $x \dot{x}=0$.

Concerning the properties of the solutions, following [OOS10], we address the issue of bounded-input-bounded-output stability. That is to say, if $g$ is bounded, is $x$ also bounded? Surprisingly, this is not always the case even if $g=0$ (see [OOS10] or Corollary 4.1). However, Theorem 4.2 states that solutions are bounded in $C^{1}-$ norm if the damping terms $\delta^{2}$ and $\omega \delta^{2}$ are not too small. Notice that this result holds for fairly general forcing term $g$. In particular, we do not suppose $g$ to be periodic.

Periodic solutions are investigated in Section 5. If $\omega=1$ then (1.1) reduces to a linear equation which admits a periodic solution. Hence we will use a perturbation argument to prove that, for $\omega \simeq 1,(1.1)$ posseses periodic solutions. This argument is based on the implicit function theorem. The problem is reduced to an algebraic equation in $\mathbb{R}^{12}$ parametrized by $\omega$. However, $g$ must be a single sinusoidal wave.

Once the existence of periodic solutions is shown, we may ask if this new WEC is more efficient than a WEC without mass modulation. From a physical point of view, we feature the following energy balance for Equation (1.1).

$$
E_{W}+E_{D}=E_{g}+E_{m}
$$


where $E_{W}$ is the energy of the WEC; $E_{g}$, the energy brought by ocean waves. $E_{D}$ is the sum of the energy harvested by the power takeoff system and the energy dissipated by hydrodynamic damping. It should be useful to distinguish between these two energies but this is not possible with the model (1.1). The new term $E_{m}$ is the energy generated by mass modulation. If the variation $\Delta E_{m}$ is positive then mass modulation increases the energy of the system. If there is no mass modulation i.e. $\omega=1$, then $\Delta E_{m}=0$.

Remark 6.1 states that, for periodic solution $x$, the variation of $E_{m}$ satisfies

$$
\Delta E_{m}:=\frac{1}{2}\left(\frac{1}{\omega}-1\right)\left(\dot{x}^{2}\left(t_{0}\right)+x^{2}\left(t_{2}\right)\right) .
$$

Thus, if $\omega<1$ then

$$
\Delta E_{D}=\Delta E_{g}+\Delta E_{m}>\Delta E_{g} .
$$

In this sense, the new WEC proposed by Orazov et al. is more efficient than standard oscillators.

\section{Mathematical Settings}

In this section, we will define solutions for Equation (1.1). We choose a continuous dynamical system approach. We will make use of the following assumptions.

$$
\begin{aligned}
& g \in C^{1}(\mathbb{R}, \mathbb{R}) \\
& g \in C^{2}(\mathbb{R}, \mathbb{R}) \\
& \text { if } \dot{g}\left(t_{0}\right)=0 \text { for some } t_{0} \in \mathbb{R} \text { then } \ddot{g}\left(t_{0}\right) \neq 0 \\
& \delta \in \mathbb{R}, \omega>0 .
\end{aligned}
$$

A generic vector in $\mathbb{R}^{3}$ will be denoted by $\left(\begin{array}{l}x \\ \dot{x} \\ t\end{array}\right)$. Setting

$$
\begin{aligned}
& A_{+}=\left\{\left(\begin{array}{l}
x \\
\dot{x} \\
t
\end{array}\right) \in \mathbb{R}^{3} \mid x \dot{x}>0\right\} \\
& A_{-}=\left\{\left(\begin{array}{l}
x \\
\dot{x} \\
t
\end{array}\right) \in \mathbb{R}^{3} \mid x \dot{x}<0\right\} \\
& A_{0}=\left\{\left(\begin{array}{l}
x \\
\dot{x} \\
t
\end{array}\right) \in \mathbb{R}^{3} \mid x \dot{x}=0\right\},
\end{aligned}
$$

we have a partition of $\mathbb{R}^{3}$, namely

$$
\mathbb{R}^{3}=A_{+} \dot{\cup} A_{-} \dot{U} A_{0} .
$$


In the sequel, we will use the following subsets of $A_{0}$.

$$
\begin{aligned}
& A_{0-+}=\left\{\left(\begin{array}{l}
0 \\
\dot{x} \\
t
\end{array}\right) \in \mathbb{R}^{3}\right\} \cup\left\{\left(\begin{array}{l}
x \\
0 \\
t
\end{array}\right) \in \mathbb{R}^{3} \mid\right. \\
& x(-x+g(t))>0 \text { or }\{-x+g(t)=0 \text { and } \dot{g}(t)=0 \text { and } x \ddot{g}(t)>0\}\} \\
& A_{0+-}=\left\{\left(\begin{array}{l}
x \\
0 \\
t
\end{array}\right) \in \mathbb{R}^{3} \mid x(-x+g(t))<0\right. \text { or } \\
& \{-x+g(t)=0 \text { and } \dot{g}(t)=0 \text { and } x \ddot{g}(t)<0\}\} \\
& A_{0++}=\left\{\left(\begin{array}{l}
x \\
0 \\
t
\end{array}\right) \in \mathbb{R}^{3} \mid x \neq 0,-x+g(t)=0, x \dot{g}(t)>0\right\} \\
& A_{0--}=\left\{\left(\begin{array}{l}
x \\
0 \\
t
\end{array}\right) \in \mathbb{R}^{3} \mid x \neq 0,-x+g(t)=0, x \dot{g}(t)<0\right\} \text {. }
\end{aligned}
$$

Roughly speaking, if a solution $x$ of (1.1) satisfies $\left(\begin{array}{c}x\left(t_{0}\right) \\ \dot{x}\left(t_{0}\right) \\ t_{0}\end{array}\right) \in A_{0-+}$ for a certain time $t_{0}$ then the curve $t \mapsto\left(\begin{array}{c}x(t) \\ \dot{x}(t) \\ t\end{array}\right)$ leaves $A_{-}$and enters $A_{+}$at time $t_{0}$. With the above notation, we have the following results. Since their proof are obvious, Proposition 2.1 and Lemma 2.2 are stated without proof.

Proposition 2.1. If $(2.2)$ and $(2.3)$ hold then $A_{0-+}, A_{0+-}, A_{0++}$ and $A_{0--}$ form a partition of $A_{0}$.

Next, we compute a Taylor expansion of $x \dot{x}$.

Lemma 2.2. If $x \in C^{4}(\mathbb{R}, \mathbb{R})$ then for $t \in \mathbb{R}$ and $h \simeq 0$, we have

$$
\begin{aligned}
x \dot{x}(t+h)=x \dot{x}(t)+\left(x \ddot{x}+\dot{x}^{2}\right)(t) h+\left(\frac{1}{2} x \dddot{x}+\right. & \left.\frac{3}{2} \dot{x} \ddot{x}\right)(t) h^{2} \\
& +\left(\frac{1}{6} x \dddot{x}+\frac{2}{3} \dot{x} \dddot{x}+\frac{1}{2} \ddot{x}^{2}\right)(t) h^{3}+O\left(h^{4}\right) .
\end{aligned}
$$

Lemma 2.3. Assume (2.2)-(2.4). Let $\left(x_{0}, \dot{x}_{0}, t_{0}\right)^{t} \in \mathbb{R}^{3}$ and $x$ be the solution of

$$
\left\{\begin{array}{l}
\ddot{x}+\omega(2 \delta \dot{x}+x-g)=0 \quad \text { on } \mathbb{R} \\
x\left(t_{0}\right)=x_{0}, \quad \dot{x}\left(t_{0}\right)=\dot{x}_{0}
\end{array}\right.
$$

For all $t \in \mathbb{R}$, we put $V(t)=(x(t), \dot{x}(t), t)^{t}$. Then, for some $\varepsilon>0$,

(1) $V\left(t_{0}\right) \in A_{0-+}$ if and only if $V\left(\left(t_{0}-\varepsilon, t_{0}\right)\right) \subset A_{-}$and $V\left(\left(t_{0}, t_{0}+\varepsilon\right)\right) \subset A_{+}$;

(2) $V\left(t_{0}\right) \in A_{0+-}$ if and only if $V\left(\left(t_{0}-\varepsilon, t_{0}\right)\right) \subset A_{+}$and $V\left(\left(t_{0}, t_{0}+\varepsilon\right)\right) \subset A_{-}$;

(3) $V\left(t_{0}\right) \in A_{+} \cup A_{0++}$ if and only if $V\left(\left(t_{0}-\varepsilon, t_{0}+\varepsilon\right) \backslash\left\{t_{0}\right\}\right) \subset A_{+}$; 
(4) $V\left(t_{0}\right) \in A_{-} \cup A_{0--}$ if and only if $V\left(\left(t_{0}-\varepsilon, t_{0}+\varepsilon\right) \backslash\left\{t_{0}\right\}\right) \subset A_{-}$.

Proof. We will first prove the if part of each assertion. If $V\left(t_{0}\right)$ belongs to $A_{+} \cup A_{-}$, the result follows from the continuity of $x \dot{x}$. If $V\left(t_{0}\right) \in A_{0++}$ then $\dot{x}\left(t_{0}\right)=0$ and $(-x+g)\left(t_{0}\right)=0$, hence

$$
\begin{aligned}
\ddot{x}\left(t_{0}\right) & =\omega(-2 \delta \dot{x}-x+g)\left(t_{0}\right)=0 \\
\dddot{x}\left(t_{0}\right) & =\omega(-2 \delta \ddot{x}-\dot{x}+\dot{g})\left(t_{0}\right)=\omega \dot{g}\left(t_{0}\right) .
\end{aligned}
$$

With Lemme 2.2,

$$
x \dot{x}\left(t_{0}+h\right)=\frac{1}{2} x \dddot{x}\left(t_{0}\right) h^{2}+O\left(h^{3}\right)=\frac{\omega}{2} x \dot{g}\left(t_{0}\right) h^{2}+O\left(h^{3}\right) .
$$

From the definition of $A_{0++}$, we have $x \dot{g}\left(t_{0}\right)>0$, thus $V\left(\left(t_{0}-\varepsilon, t_{0}+\varepsilon\right) \backslash\left\{t_{0}\right\}\right) \subset A_{+}$.

The proof for $V\left(t_{0}\right) \in A_{0--}$ is analog. If $V\left(t_{0}\right) \in A_{0-+}$ then one of the four cases below holds.

1) $x\left(t_{0}\right)=0, \dot{x}\left(t_{0}\right) \neq 0$. By Lemma 2.2 ,

$$
x \dot{x}\left(t_{0}+h\right)=\dot{x}\left(t_{0}\right)^{2} h+O\left(h^{2}\right) .
$$

Hence, there exists some positive $\varepsilon$ such that $V\left(\left(t_{0}-\varepsilon, t_{0}\right)\right) \subset A_{-}$and $V\left(\left(t_{0}, t_{0}+\varepsilon\right)\right) \subset$ $A_{+}$.

2) $x\left(t_{0}\right) \neq 0, \dot{x}\left(t_{0}\right)=0$ and $x(-x+g)\left(t_{0}\right)>0$. Then

$$
\begin{aligned}
\ddot{x}\left(t_{0}\right) & =\omega(-x+g)\left(t_{0}\right) \\
x \dot{x}\left(t_{0}+h\right) & =\omega x(-x+g)\left(t_{0}\right) h+O\left(h^{2}\right) .
\end{aligned}
$$

And we obtain the same conclusion as in the previous case since $\omega>0$.

3) $x\left(t_{0}\right) \neq 0, \dot{x}\left(t_{0}\right)=0,(-x+g)\left(t_{0}\right)=\dot{g}\left(t_{0}\right)=0$ and $x \ddot{g}\left(t_{0}\right)>0$. Then

$$
\begin{aligned}
\ddot{x}\left(t_{0}\right) & =\dddot{x}\left(t_{0}\right)=0 \\
\dddot{x}\left(t_{0}\right) & =\omega(-2 \delta \dddot{x}-\ddot{x}+\ddot{g})\left(t_{0}\right)=\omega \ddot{g}\left(t_{0}\right) .
\end{aligned}
$$

With Lemma 2.2,

$$
x \dot{x}\left(t_{0}+h\right)=\frac{1}{6} x \dddot{x}\left(t_{0}\right) h^{3}+O\left(h^{4}\right)=\frac{\omega}{6} x \ddot{g}\left(t_{0}\right) h^{3}+O\left(h^{4}\right) .
$$

And the same conclusion holds.

4) $x\left(t_{0}\right)=0, \dot{x}\left(t_{0}\right)=0$. Then

$$
\begin{aligned}
\ddot{x}\left(t_{0}\right) & =\omega g\left(t_{0}\right) \\
x \dot{x}(t+h) & =\frac{1}{2} \ddot{x}^{2}\left(t_{0}\right) h^{3}+O\left(h^{4}\right)=\frac{1}{2} \omega^{2} g^{2}\left(t_{0}\right) h^{3}+O\left(h^{4}\right) .
\end{aligned}
$$

If $g\left(t_{0}\right) \neq 0$ then we are done. If $g\left(t_{0}\right)=0$ then

$$
\dddot{x}\left(t_{0}\right)=\omega(-2 \delta \ddot{x}-\dot{x}+\dot{g})\left(t_{0}\right)=\omega \dot{g}\left(t_{0}\right) .
$$

If $\dot{g}\left(t_{0}\right)>0$ then $\dddot{x}\left(t_{0}\right)>0$ and we can deduce the sign of $x \dot{x}$ from the variations of $x$ and $\dot{x}$. Indeed, since $\ddot{x}\left(t_{0}\right)=0, \ddot{x}$ is negative on some interval of the form $\left(t_{0}-\varepsilon, t_{0}\right)$ and positive on $\left(t_{0}, t_{0}+\varepsilon\right)$. Hence $\dot{x}$ is positive in a neighborhood of $t_{0}$ except in $t_{0}$ where it vanishes. Finally, we obtain that $x$ is negative on some interval of the form $\left(t_{0}-\varepsilon, t_{0}\right)$ and positive on $\left(t_{0}, t_{0}+\varepsilon\right)$. If $\dot{g}\left(t_{0}\right)<0$ then all signs are reversed and we have the same conclusion. 
If $\dot{g}\left(t_{0}\right)=0$ then $\ddot{g}\left(t_{0}\right) \neq 0$ according to $(2.3)$. Then

$$
\begin{aligned}
& x=\dot{x}=\ddot{x}=\dddot{x}=0 \quad \text { at } t_{0} \\
& \dddot{x}\left(t_{0}\right)=\omega \ddot{g}\left(t_{0}\right) \neq 0 .
\end{aligned}
$$

As in the case $\dot{g}\left(t_{0}\right) \neq 0$, we compute the sign of $x \dot{x}$ by studying the variations of $x$ and $\dot{x}$. However, we consider at first, $\dddot{x}$.

The four cases above prove the if part of the assertion (1) in Lemma 2.3, namely

$$
V\left(t_{0}\right) \in A_{0-+} \Longrightarrow V\left(\left(t_{0}-\varepsilon, t_{0}\right)\right) \subset A_{-}, V\left(\left(t_{0}, t_{0}+\varepsilon\right)\right) \subset A_{+} .
$$

The case where $V\left(t_{0}\right) \in A_{0+-}$ is proved in the same way, which conclude the if part of the proof.

Conversely, assume that $V\left(\left(t_{0}-\varepsilon, t_{0}\right)\right) \subset A_{-}$and $V\left(\left(t_{0}, t_{0}+\varepsilon\right)\right) \subset A_{+}$for some $\varepsilon>0$. Then $V\left(t_{0}\right)$ cannot belong to $A_{0+-} \cup A_{+} \cup A_{0++} \cup A_{-} \cup A_{0--}$ according to the if part of the proof. Hence $V\left(t_{0}\right)$ is in $A_{0-+}$ by Proposition 2.1. This proves the equivalence (1) of the lemma. The other cases are proved in the same way, which completes the proof of the Lemma.

\subsection{Solutions of (1.1) and the corresponding Cauchy Problem.}

Definition 2.1 (Local solutions). Let $I$ be an interval of $\mathbb{R}$. Under assumptions (2.1) and (2.4), we say that $x$ is a local solution to (1.1) on $I$ if

- $x \in C^{1}(I, \mathbb{R})$ and $I$ has positive (Lebesgue) measure;

- one of the three conditions below holds.

(i) $x \in C^{2}(I, \mathbb{R}), V(t):=(x(t), \dot{x}(t), t)^{t}$ belongs to $A_{+} \cup A_{0++}$ for all $t \in I$ and

$$
\ddot{x}+2 \omega \delta \dot{x}+\omega x=\omega g \quad \text { on } I .
$$

(ii) $x \in C^{2}(I, \mathbb{R}), V(t)$ belongs to $A_{-} \cup A_{0--}$ for all $t \in I$ and

$$
\ddot{x}+2 \delta \dot{x}+x=g \quad \text { on } I .
$$

(iii) There is a time $t_{0}$ in $I$ such that if $I_{1}:=I \cap\left(-\infty, t_{0}\right)$ and $I_{2}:=I \cap\left(t_{0}, \infty\right)$ then one of the two following conditions holds.

(iii-a) $V\left(I_{1}\right) \subset A_{-}, V\left(I_{2}\right) \subset A_{+}$and

$$
\begin{array}{lll}
\ddot{x}+2 \omega \delta \dot{x}+\omega x=\omega g & \text { on } I_{2} \\
\ddot{x}+2 \delta \dot{x}+x=g & \text { on } I_{1} .
\end{array}
$$

(iii-b) $V\left(I_{1}\right) \subset A_{+}, V\left(I_{2}\right) \subset A_{-}$and

$$
\begin{array}{lll}
\ddot{x}+2 \omega \delta \dot{x}+\omega x=\omega g & \text { on } I_{1} \\
\ddot{x}+2 \delta \dot{x}+x=g & \text { on } I_{2} .
\end{array}
$$

Remark 2.1. This definition excludes oscillatory solutions $x$ such that $x \dot{x}$ has infinetly many changes of signs on some compact interval. For instance, solutions of the form $x(t)=t^{5} \sin \frac{1}{t}$ are excluded.

If $x$ is a local solution on $I$ then $x \dot{x}$ changes its sign at most once (case (iii)). However, $x \dot{x}$ may have many zeros on $I$. Indeed, in case (i), if $V(t)$ belongs to $A_{0++}$ then $x \dot{x}(t)=0$. 
If (2.2)-(2.4) hold and $I$ is an open interval then, in case (iii-a), $V\left(t_{0}\right)$ lies in $A_{0-+}$ and in case (iii-b), $V\left(t_{0}\right)$ lies in $A_{0+-}$ (see Lemma 2.3).

If $g=0$ then $x=0$ is not a local solution since $0 \in A_{0-+}$.

Definition 2.2. Let $J$ be an interval of $\mathbb{R}$. We say that $x$ is a (global) solution of (1.1) on $J$ if

- $x \in C^{1}(J, \mathbb{R})$;

- for all $t_{0} \in J$, there exists a interval $I \subset J$, relatively open in $J$ and containing $t_{0}$ such that $x$ is a local solution on $I$.

Definition 2.3. Let $J$ be an interval of $\mathbb{R}, t_{0} \in J$ and $x_{0}, \dot{x}_{0}$ be two real numbers. Under assumptions (2.1) and (2.4), we say that $x$ is a solution to the Cauchy problem (corresponding to (1.1)) with initial condition $\left(x_{0}, \dot{x}_{0}\right)$ at time $t_{0}$ if

- $x$ is a global solution of (1.1) on $J$;

- $x\left(t_{0}\right)=x_{0}$ and $\dot{x}\left(t_{0}\right)=\dot{x}_{0}$.

\section{Existence and Uniqueness of the CAUChy PRoblem}

In this section, we will consider separately the cases where the forcing term $g$ is non trivial and the case where it is identically equal to zero.

Theorem 3.1. Assume (2.2)-(2.4) and

$$
g(t)=0 \Longrightarrow \dot{g}(t) \neq 0 .
$$

Then, for any $t_{0}, x_{0}$ and $\dot{x}_{0}$ in $\mathbb{R}$, the Cauchy problem corresponding to (1.1) with initial condition $\left(x_{0}, \dot{x}_{0}\right)$ at time $t_{0}$ admits a unique solution on $\mathbb{R}$.

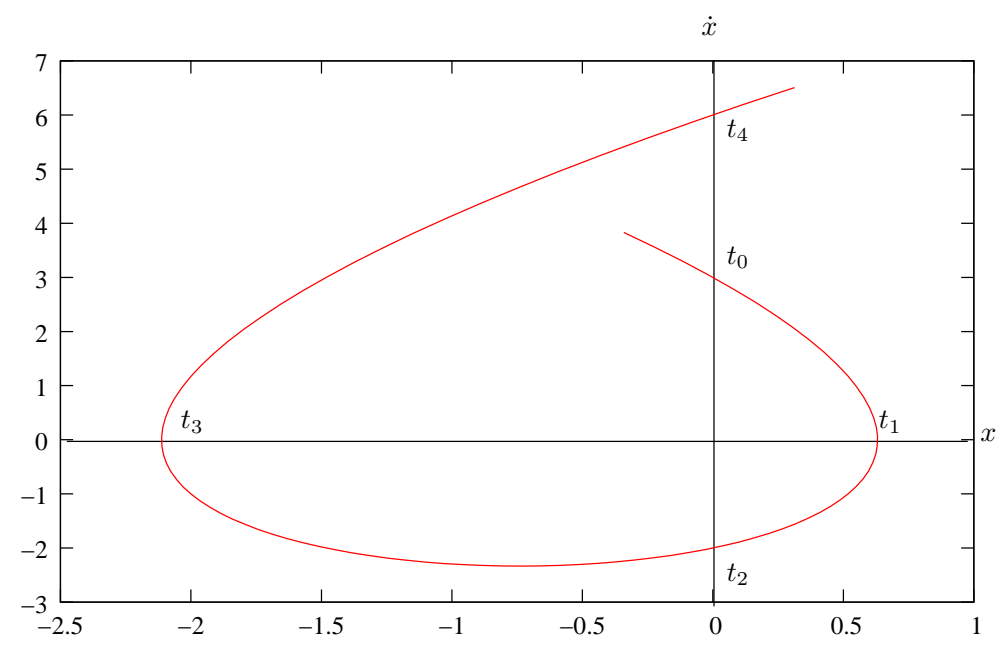

Figure 1 . Trajectory in the phase space $(x, \dot{x})$ for a positive cycle, in the sense of Definition 6.1. 
Proof. Uniqueness. Let $x$ and $y$ two solutions of the above Cauchy problem and suppose that $(x, \dot{x})(t) \neq(y, \dot{y})(t)$ for some time $t>t_{0}$. If $t_{1}$ denotes the infimum of these $t$ then $(x, \dot{x})\left(t_{1}\right)=(y, \dot{y})\left(t_{1}\right)$ and there exists a decreasing sequence $\left(\tau_{n}\right)_{n \geq 0} \subset \mathbb{R}$ such that

$$
\tau_{n} \underset{n \rightarrow \infty}{\longrightarrow} t_{1} \text { and }(x, \dot{x})\left(\tau_{n}\right) \neq(y, \dot{y})\left(\tau_{n}\right), \forall n \geq 0 .
$$

Since $x, y$ are global solutions, there exists some $\varepsilon>0$ such that $x$ and $y$ are local solutions on $I:=\left(t_{1}-\varepsilon, t_{1}+\varepsilon\right)$; here we have used the fact that, in Definition $2.2, I$ is relatively open in $J=\mathbb{R}$. According to (3) Lemma 2.3, if $V\left(t_{1}\right) \in A_{+} \cup A_{0++}$ then $x$ and $y$ satisfy condition (i) of Definition 2.1 on $\left(t_{1}-\varepsilon^{\prime}, t_{1}+\varepsilon^{\prime}\right)$, for some $\varepsilon^{\prime} \in(0, \varepsilon]$. Hence $x \equiv y$ on $I$ by Cauchy's Theorem. We then get a contradiction with (3.2).

Cases (1), (2) and (4) of Lemma 2.3 lead also to a contradiction. Then Proposition 2.1 warrants that there is no other possibility which finish to prove the uniqueness of the solution to the Cauchy problem.

Existence. We will prove that there exists a solution on $\left[t_{0}, \infty\right)$. Performing the same construction on $\left(-\infty, t_{0}\right)$, we will obtain a global solution on $\mathbb{R}$.

If $V\left(t_{0}\right) \in A_{+} \cup A_{0-+} \cup A_{0++}$ then let $x$ be the solution of

$$
\begin{aligned}
& \ddot{x}+\omega(2 \delta \dot{x}+x-g)=0 \quad \text { on }\left[t_{0}, \infty\right) \\
& x\left(t_{0}\right)=x_{0}, \quad \dot{x}\left(t_{0}\right)=\dot{x}_{0} .
\end{aligned}
$$

By Lemma 2.3, $x \dot{x}>0$ on $\left(t_{0}, t_{0}+\varepsilon\right)$ for some positive $\varepsilon$. If $x \dot{x} \geq 0$ on $\left[t_{0}, \infty\right)$ then we have a solution on $\left[t_{0}, \infty\right)$. Otherwise, there is a time $t_{1}>t_{0}$ such that

$$
x \dot{x}>0 \text { on }\left[t_{0}, t_{1}\right), \quad\left(x\left(t_{1}\right), \dot{x}\left(t_{1}\right), t_{1}\right)^{t} \in A_{0+-} .
$$

Then $x_{\mid\left[t_{0}, t_{1}\right)}$ is a local solution on $\left[t_{0}, t_{1}\right)$.

If $V\left(t_{0}\right) \in A_{-} \cup A_{0+-} \cup A_{0--}$ then we consider the solution $x$ of

$$
\begin{aligned}
& \ddot{x}+2 \delta \dot{x}+x-g=0 \quad \text { on }\left[t_{0}, \infty\right) \\
& x\left(t_{0}\right)=x_{0}, \quad \dot{x}\left(t_{0}\right)=\dot{x}_{0} .
\end{aligned}
$$

We argue as in the previous case to get a local solution on $\left[t_{0}, t_{1}\right)$ for some $t_{1} \in\left(t_{0}, \infty\right]$.

Without loss of generality, we may assume $V\left(t_{0}\right) \in A_{+} \cup A_{0-+} \cup A_{0++}$ and $t_{1}<\infty$. Then by induction, we construct an increasing sequence of time $t_{1}, \ldots, t_{N}, t_{N+1}$ with $N \geq 1, t_{N} \in \mathbb{R}$ and $t_{N+1} \in\left(t_{N}, \infty\right]$ so that we have a global solution on $\left[t_{0}, t_{N+1}\right)$. If $t_{N+1}=\infty$ then we have a solution on $\left[t_{0}, \infty\right)$. Otherwise, there exists an increasing sequence $\left(t_{n}\right)_{n \geq 0}$ and $x$ in $C^{1}\left(\cup_{n \geq 0}\left[t_{n}, t_{n+1}\right], \mathbb{R}\right)$ such that

$$
\begin{array}{ll}
V\left(t_{2 n}\right) \in A_{0-+}, \quad V\left(t_{2 n-1}\right) \in A_{0+-} & \forall n \geq 1 \\
x \in C^{2}\left(\left(t_{n}, t_{n+1}\right), \mathbb{R}\right) & \\
\ddot{x}+\omega(2 \delta \dot{x}+x-g)=0, \quad x \dot{x} \geq 0 & \text { on }\left(t_{2 n}, t_{2 n+1}\right) \\
\ddot{x}+2 \delta \dot{x}+x-g=0, \quad x \dot{x} \leq 0 & \text { on }\left(t_{2 n+1}, t_{2 n+2}\right), \forall n \geq 0 .
\end{array}
$$


Then the theorem will be proved if $t_{n} \rightarrow \infty$. Let us argue by contradiction assuming that $t_{n} \rightarrow T \in \mathbb{R}$. Then we denote by $a$ the function of $L^{\infty}(\mathbb{R})$ defined by

$$
a(x)= \begin{cases}\omega & \text { if } x>0 \\ 1 & \text { if } x<0 .\end{cases}
$$

Since $\dot{x}$ is continuous on $\left[t_{0}, T\right)$ and $x \dot{x}$ vanishes on a set measure zero (see Lemma 2.3), we have for all $t \in\left[t_{0}, T\right)$,

$$
\dot{x}(t)-\dot{x}\left(t_{0}\right)+\int_{t_{0}}^{t} a(x \dot{x})(2 \delta \dot{x}+x-g)(s) \mathrm{d} s=0 .
$$

Then

$$
\begin{aligned}
& |\dot{x}(t)| \leq\left|\dot{x}\left(t_{0}\right)\right|+\max (1, \omega) \int_{t_{0}}^{t} 2|\delta||\dot{x}|+|x|+|g| \mathrm{d} s \\
& |x(t)| \leq\left|x\left(t_{0}\right)\right|+\int_{t_{0}}^{t}|\dot{x}| \mathrm{d} s .
\end{aligned}
$$

Hence, $y(t):=|x(t)|+|\dot{x}(t)|$ satisfies

$$
y(t) \leq y\left(t_{0}\right)+C\left(T-t_{0}\right) \sup _{\left[t_{0}, T\right)}|g|+C \int_{t_{0}}^{t} y(s) \mathrm{d} s
$$

where $C$ is a constant depending only on $\delta$ and $\omega$. By Gronwall's Lemma, $y$ is bounded on $\left[t_{0}, T\right)$. Thus, using also (3.7) with $t_{0}=t^{\prime}$, we obtain for another constant still labelled $C$,

$$
\begin{aligned}
& \left|x(t)-x\left(t^{\prime}\right)\right| \leq C\left|t-t^{\prime}\right| \\
& \left|\dot{x}(t)-\dot{x}\left(t^{\prime}\right)\right| \leq C\left|t-t^{\prime}\right| \quad \forall t, t^{\prime} \in\left[t_{0}, T\right) .
\end{aligned}
$$

Hence, there exist $a, b$ in $\mathbb{R}$ such that

$$
x(t) \rightarrow a, \quad \dot{x}(t) \rightarrow b \quad \text { as } t \rightarrow T .
$$

By (3.4), $V\left(t_{2 n+1}\right) \in A_{0+-}$ thus $\dot{x}\left(t_{2 n+1}\right)=0$ and $b=0$.

If $a \neq g(T)$ then for all $t \in \cup_{n \geq 0}\left(t_{2 n}, t_{2 n+1}\right)$,

$$
\ddot{x}(t)=\omega(-2 \delta \dot{x}-x+g)(t) \underset{t \rightarrow T}{\longrightarrow} \omega(-a+g(T)) \neq 0 .
$$

Since $\omega>0$, we deduce that $\ddot{x}$ has a sign on $\cup_{n \geq n_{1}}\left(t_{n}, t_{n+1}\right)$ for some large index $n_{1}$. Then $\dot{x}$ is monotone (since it is continuous) on $[T-\varepsilon, T[$. Thus $x \dot{x}$ has a sign on $\left[T-\varepsilon^{\prime}, T[\right.$ which contradicts (3.5) and (3.6).

Then $a=g(T)$. From (3.5), (3.6), we deduce

$$
\lim _{t \rightarrow T, t \in \cup_{n \geq 0}\left(t_{n}, t_{n+1}\right)} \ddot{x}(t)=0 .
$$

Differentiating (3.5) and (3.6) with respect to time, we get

$$
\lim _{t \rightarrow T, t \in \cup_{n \geq 0}\left(t_{2 n}, t_{2 n+1}\right)} \dddot{x}(t)=\omega \dot{g}(T), \quad \lim _{t \rightarrow T, t \in \cup_{n \geq 0}\left(t_{2 n+1}, t_{2 n+2}\right)} \dddot{x}(t)=\dot{g}(T) .
$$


If $\dot{g}(T) \neq 0$ then $\dddot{x}$ has a sign on $\cup_{n \geq n_{0}}\left(t_{n}, t_{n+1}\right)$ for some large index $n_{0}$. Consequently $\ddot{x}$ is monotone on $\cup_{n \geq n_{0}}\left(t_{n}, t_{n+1}\right)$. Remark that since $\ddot{x}$ is not continuous, we cannot deduce, at this stage, that $\ddot{x}$ has a sign in a left neighborhood of $T$. However we may obtain the sign of $x \dot{x}$ with Lemma 3.2 below. Now we will check that the last assumption of Lemma 3.2 holds.

By (3.5), (3.6),

$$
\ddot{x}\left(t_{2 n+1}^{-}\right)=\omega \ddot{x}\left(t_{2 n+1}^{+}\right), \quad \ddot{x}\left(t_{2 n}^{+}\right)=\omega \ddot{x}\left(t_{2 n}^{-}\right)
$$

where

$$
\ddot{x}\left(t_{2 n+1}^{-}\right):=\lim _{t \rightarrow t_{2 n+1}, t<t_{2 n+1}} \ddot{x}(t) .
$$

Since $\omega>0$, we have $\ddot{x}\left(t_{n}^{-}\right) \ddot{x}\left(t_{n}^{+}\right) \geq 0$. Then Lemma 3.2 leads to a contradiction with (3.5) and (3.6).

Lemma 3.2. Let $t_{0}, T$ be real numbers such that $t_{0}<T$. Assume that $\left(t_{n}\right)_{n \geq 0} \subset \mathbb{R}$ increases toward $T$. Besides, let $x \in W^{2, \infty}\left(t_{0}, T\right)$ be such that

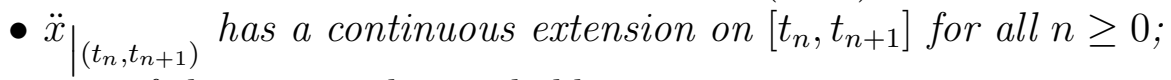

- one of the two conditions holds:

(i) $\ddot{x}$ is increasing on $\left(t_{n}, t_{n+1}\right)$, for all $n \geq 0$; or

(ii) $\ddot{x}$ is decreasing on $\left(t_{n}, t_{n+1}\right)$, for all $n \geq 0$;

- $\ddot{x}\left(t_{n}^{-}\right) \ddot{x}\left(t_{n}^{+}\right) \geq 0$ for all $n \geq 1$.

Then $x \dot{x}$ has a sign on $(T-\varepsilon, T)$ some some positive $\varepsilon$, i.e. $x \dot{x}>0$ on $(T-\varepsilon, T)$ or $x \dot{x}<0$ on $(T-\varepsilon, T)$.

Proof. If $\ddot{x}$ is increasing on $\left(t_{n}, t_{n+1}\right)$ for all $n \geq 0$ then one of the two following cases holds.

1) $\ddot{x}\left(t_{n_{1}}^{-}\right)>0$ for some index $n_{1} \geq 1$. Then $\ddot{x}\left(t_{n_{1}}^{+}\right) \geq 0$ since $\ddot{x}\left(t_{n}^{-}\right) \ddot{x}\left(t_{n}^{+}\right) \geq 0$ by assumption. Thus $\ddot{x}\left(t_{n_{1}+1}^{-}\right)>0$ since $\ddot{x}$ is increasing on $\left(t_{n_{1}}, t_{n_{1}+1}\right)$. By induction, $\ddot{x}\left(t_{n}^{-}\right)>0$ for all $n \geq n_{1}$. Thus, for all $n \geq n_{1}, \ddot{x}\left(t_{n}^{+}\right) \geq 0$ (since $\ddot{x}\left(t_{n}^{-}\right) \ddot{x}\left(t_{n}^{+}\right) \geq 0$ ) and, then, $\ddot{x}>0$ on $\left(t_{n}, t_{n+1}\right)$. Hence $\dot{x}$ is increasing on $\left(t_{n_{1}}, T\right)$ and has a sign since it is continuous. We then deduce that $x \dot{x}$ has a sign on $(T-\varepsilon, T)$.

2) $\ddot{x}\left(t_{n}^{-}\right) \leq 0$ for all $n \geq 1$. Hence $\ddot{x}<0$ on $\left(t_{n}, t_{n+1}\right)$ for all $n \geq 0$, since $\ddot{x}$ is increasing. Thus $\ddot{x}<0$ a.e. on $\left(t_{0}, T\right)$. Then $\dot{x}$ is decreasing. As above, we conclude that $x \dot{x}$ has a sign on $(T-\varepsilon, T)$.

The case where $\ddot{x}$ is decreasing is analog.

Let us continue the proof of Theorem 3.1. There remains to consider the case where $\dot{g}(T)=0$. Then $\ddot{g}(T) \neq 0$ by (2.3). We obtain from (3.5), (3.6) and (3.9),

$$
\dddot{x}(t)=\left\{\begin{array}{ll}
\omega(-2 \delta \dddot{x}-\ddot{x}+\ddot{g})(t) \rightarrow \omega \ddot{g}(T) & \text { if } t \in \cup_{n \geq 0}\left(t_{2 n}, t_{2 n+1}\right) \\
(-2 \delta \dddot{x}-\ddot{x}+\ddot{g})(t) \rightarrow \ddot{g}(T) & \text { if } t \in \cup_{n \geq 0}\left(t_{2 n+1}, t_{2 n+2}\right)
\end{array} .\right.
$$

If $\ddot{g}(T)>0$ (the case $\ddot{g}(T)<0$ can be done in a same way) then $\dddot{x}$ is increasing on $\cup_{n \geq n_{0}}\left(t_{n}, t_{n+1}\right)$ by (3.11). Let us show that $\dddot{x}<0$ on $J:=\cup_{n \geq n_{1}}\left(t_{n}, t_{n+1}\right)$ for some 
large index $n_{1}$. For this, we will first show that $\dddot{x}\left(t_{2 n+1}^{-}\right)<0$. Indeed,

$$
\begin{aligned}
& \dddot{x}\left(t_{2 n+1}^{-}\right)=\omega\left(-2 \delta \ddot{x}\left(t_{2 n+1}^{-}\right)+(-\dot{x}+\dot{g})\left(t_{2 n+1}\right)\right) \\
& \ddot{x}\left(t_{2 n+1}^{-}\right)=\omega(-x+g)\left(t_{2 n+1}\right),
\end{aligned}
$$

since $\dot{x}\left(t_{2 n+1}\right)=0$. On $J$, we have when $t \rightarrow T$,

$$
-x+g \rightarrow 0, \quad-\dot{x}+\dot{g} \rightarrow 0, \quad-\ddot{x}+\ddot{g} \rightarrow \ddot{g}(T)>0,
$$

by (3.8). Thus $-\ddot{x}+\ddot{g}>0$ a.e. on $(T-\varepsilon, T)$ for some $\varepsilon>0$. Hence,

$$
-\dot{x}+\dot{g}<0, \quad-x+g>0 \quad \text { on }(T-\varepsilon, T) .
$$

Thus, for large $n, \ddot{x}\left(t_{2 n+1}^{-}\right)>0$ (see $\left.(3.13)\right)$ and $(-\dot{x}+\dot{g})\left(t_{2 n+1}\right)<0$. With (3.12),

$$
\dddot{x}\left(t_{2 n+1}^{-}\right)<0 \text {. }
$$

Next, let us show that $\dddot{x}\left(t_{2 n}^{-}\right)<0$. We have

$$
\begin{aligned}
& \dddot{x}\left(t_{2 n}^{-}\right)=-2 \delta \ddot{x}\left(t_{2 n}^{-}\right)+(-\dot{x}+\dot{g})\left(t_{2 n}\right) \\
& \ddot{x}\left(t_{2 n}^{-}\right)=(-2 \delta \dot{x}-x+g)\left(t_{2 n}\right) .
\end{aligned}
$$

If there exists a subsequence $\left(t_{2 n_{k}}\right)_{k \geq 0}$ of $\left(t_{2 n}\right)_{n \geq 0}$ such that $\dot{x}\left(t_{2 n_{k}}\right)>0$ for all $k$ then, since $V\left(t_{2 n_{k}}\right) \in A_{0-+}($ by $(3.4))$, we have $x\left(t_{2 n_{k}}\right)=0$. Therefore $x(t) \rightarrow 0$ and $g(T)=0$. Since, it is assumed here that $\dot{g}(T)=0$, there is a contradiction with (3.1).

Thus $\dot{x}\left(t_{2 n}\right) \leq 0$ for $n$ large enough. Then (3.17), (3.14) imply that $\ddot{x}\left(t_{2 n}^{-}\right)>0$ and, with (3.14), (3.16), we obtain $\dddot{x}\left(t_{2 n}^{-}\right)<0$, and with (3.15), $\dddot{x}\left(t_{n}^{-}\right)<0$ for $n$ large enough. Since $\dddot{x}$ is increasing on $J$, we have $\dddot{x}<0$ on $J$. It follows from Lemma 3.2 that $x \dot{x}$ has a sign on $(T-\varepsilon, T)$. This contradicts again (3.5), (3.6) and completes the proof of the theorem.

3.1. The homogenous problem. In this subsection, we will assume that

$$
g=0 \quad \text { on } \mathbb{R} .
$$

Then the subsets of $A_{0}$ reduce to

$$
A_{0-+}=\left\{\left(\begin{array}{l}
0 \\
\dot{x} \\
t
\end{array}\right) \in \mathbb{R}^{3}\right\}, \quad A_{0+-}=\left\{\left(\begin{array}{l}
x \\
0 \\
t
\end{array}\right) \in \mathbb{R}^{3} \mid x \neq 0\right\}, \quad A_{0++}=A_{0--}=\emptyset .
$$

Moreover we have the following partition of $\mathbb{R}^{3}$ :

$$
\mathbb{R}^{3}=A_{+} \dot{U} A_{-} \dot{U} A_{0-+} \dot{U} A_{0+-} .
$$

Proposition 3.3. Under assumptions (2.4), (3.18), let $x$ be a global solution of (1.1) on $\mathbb{R}$. Then

$$
(x(t), \dot{x}(t)) \neq(0,0) \quad \forall t \in \mathbb{R} .
$$

Proof. Arguing by contradiction, we suppose that there exists some $t_{0}$ such that

$$
V\left(t_{0}\right)=\left(0,0, t_{0}\right)^{t} .
$$


There results that $V\left(t_{0}\right)$ belongs to $A_{0-+}$. Thus the case (iii) of Definition 2.1 holds. If (iii-b) is true then, for some positive $\varepsilon, x$ solves the problem

$$
\begin{aligned}
& \ddot{x}+2 \delta \dot{x}+x=0 \quad \text { on }\left(t_{0}, t_{0}+\varepsilon\right) \\
& x\left(t_{0}\right)=0, \quad \dot{x}\left(t_{0}\right)=0 .
\end{aligned}
$$

Hence $x=0$ on $\left(t_{0}, t_{0}+\varepsilon\right)$. But $V\left(\left(t_{0}, t_{0}+\varepsilon\right)\right) \subset A_{-}$by Definition 2.1. We get a contradiction. The case (iii-a) is analog; accordingly

$$
(x(t), \dot{x}(t)) \neq(0,0) \quad \forall t \in \mathbb{R} .
$$

Theorem 3.4. Under assumptions (2.4), (3.18), let $t_{0} \in \mathbb{R}$ and $\left(x_{0}, \dot{x}_{0}\right) \in \mathbb{R}^{2} \backslash\{(0,0)\}$. Then the Cauchy problem corresponding to (1.1) with initial condition $\left(x_{0}, \dot{x}_{0}\right)$ at time $t_{0}$ admits a unique solution.

Proof. The proof is similar and even simpler than the one of Theorem 3.1. So we skip the details.

Uniqueness. We consider two solutions $x$ and $y$ and a time $t_{1}$ satisfying $(x, \dot{x})\left(t_{1}\right)=$ $(y, \bar{y})\left(t_{1}\right)$ and $(3.2)$. Lemma 2.3 holds except the first equivalence which becomes

$$
V\left(t_{0}\right) \in A_{0-+} \backslash\left\{\left(0,0, t_{0}\right)^{t}\right\} \Leftrightarrow V\left(\left(t_{0}-\varepsilon, t_{0}\right)\right) \subset A_{-} \text {and } V\left(\left(t_{0}, t_{0}+\varepsilon\right)\right) \subset A_{+} .
$$

By Proposition 3.3,

$$
\left(x\left(t_{1}\right), \dot{x}\left(t_{1}\right), t_{1}\right)^{t} \neq\left(0,0, t_{1}\right)^{t},
$$

so that the uniqueness follows as in Theorem 3.1.

Existence. For the homogenous equation, explicit solutions on $A_{+}$and $A_{-}$are available. We then construct a solution using the method of Theorem 3.1.

We will now investigate some properties of oscillating solutions of (1.1) when $g=0$. They are produced when $\delta$ and $\omega \delta^{2}$ range $(0,1)$.

Proposition 3.5. Let us assume that $\delta$ and $\omega \delta^{2}$ belong to $(0,1)$ and $g=0$. If $x$ denotes the solution given by Theorem 3.4 then there exits a time $\tau_{0} \in \mathbb{R}$ such that $x\left(\tau_{0}\right)=0$ and $\dot{x}\left(\tau_{0}\right)>0$.

Proof. Arguing by contradiction, we assume that

$$
x(t) \neq 0 \quad \text { or } \quad \dot{x}(t) \leq 0, \quad \forall t \in \mathbb{R} .
$$

Then there exists a time $t_{0}$ such that

$$
x<0 \quad \text { in }\left(t_{0}, \infty\right) \quad \text { or } \quad x>0 \text { in }\left(t_{0}, \infty\right) .
$$

Indeed, if $x\left(t_{0}\right)=0$ then (3.19) and Proposition 3.3 imply $x<0$ in $\left(t_{0}, \infty\right)$; hence (3.20) follows.

Next, we set

$$
y= \begin{cases}x & \text { if } x>0 \text { in }\left(t_{0}, \infty\right) \\ -x & \text { if } x<0 \text { in }\left(t_{0}, \infty\right) .\end{cases}
$$

Then $y$ solves $(1.1)$ and $y>0$ in $\left(t_{0}, \infty\right)$. We claim that, for some $t_{1} \geq t_{0}$,

$$
\dot{y}<0 \quad \text { in }\left(t_{1}, \infty\right) \quad \text { or } \quad \dot{y} \geq 0 \quad \text { in }\left(t_{1}, \infty\right) \text {. }
$$


Indeed, if $\dot{y}\left(t_{1}\right)<0$ and there exists $t_{2}>t_{1}$ such that

$$
\dot{y}\left(t_{2}\right)=0, \quad \dot{y}<0 \quad \text { on }\left[t_{1}, t_{2}\right),
$$

then $\ddot{y}\left(t_{2}^{-}\right) \geq 0$; recall that $\ddot{y}\left(t_{2}^{-}\right):=\lim _{t \rightarrow t_{2}, t<t_{2}} \ddot{y}(t)$. However,

$$
\underbrace{\ddot{y}\left(t_{2}^{-}\right)}_{\geq 0}+2 \omega \delta \underbrace{\dot{y}\left(t_{2}\right)}_{=0}+\underbrace{y\left(t_{2}\right)}_{>0}=0 .
$$

We get a contradiction, therefore, (3.21) follows.

Using $y>0$ in $\left(t_{0}, \infty\right)$ and $(3.21)$, we deduce that $y$ is monotone in $\left(t_{1}, \infty\right)$ and satisfies

$$
\ddot{y}+2 \delta \dot{y}+y=0 \quad \text { in }\left(t_{1}, \infty\right) \quad \text { or } \quad \ddot{y}+2 \omega \delta \dot{y}+\omega y=0 \quad \text { in }\left(t_{1}, \infty\right) .
$$

By computing the explicit solution, we obtain a contradiction since $\delta$ and $\omega \delta^{2}$ belong to $(0,1)$. This completes the proof of the proposition.

The next result is due to Orazov et al.

Proposition 3.6 ([OOS10]). Let us assume that $\delta$ and $\omega \delta^{2}$ belong to $(0,1)$ and $g=0$. For all global solution $x$ of (1.1), there exists an increasing sequence of time $\left(t_{n}\right)_{n \geq 0}$ such that the following properties hold for all $n \geq 0$.

(1) If $u:=(x, \dot{x})^{t}$ then

$$
\begin{array}{ll}
u\left(t_{4 n}\right) \in\{0\} \times(0, \infty) & u\left(t_{4 n+1}\right) \in(0, \infty) \times\{0\} \\
u\left(t_{4 n+2}\right) \in\{0\} \times(-\infty, 0) & u\left(t_{4 n+3}\right) \in(-\infty, 0) \times\{0\} .
\end{array}
$$

(2) $x \dot{x}>0$ in $\left(t_{2 n}, t_{2 n+1}\right)$ and $x \dot{x}<0$ in $\left(t_{2 n+1}, t_{2 n+2}\right)$.

(3) With the notation

$$
\begin{aligned}
\Omega_{\omega} & :=\sqrt{\omega}\left(1-\omega \delta^{2}\right)^{1 / 2} \\
T_{\omega} & :=\frac{1}{\Omega_{\omega}} \arctan \frac{\Omega_{\omega}}{\omega \delta} \\
T_{-} & :=\frac{\pi}{\Omega_{1}}-\frac{1}{\Omega_{1}} \arctan \frac{\Omega_{1}}{\delta},
\end{aligned}
$$

we have

$$
t_{n+2}-t_{n}=t_{2}-t_{0}=T_{\omega}+T_{-} .
$$

(4) Finally, by setting

$$
k(\omega):=\exp \left(-\omega \delta T_{\omega}-\delta T_{-}\right) \frac{1}{\sqrt{\omega}},
$$

there holds

$$
\dot{x}\left(t_{4 n+2}\right)=-k(\omega) \dot{x}\left(t_{4 n}\right) .
$$

Proof. By Proposition 3.3, $V(0) \neq 0$. By Proposition 3.5, we may assume without loss of generality, that $u(0)$ lies in $\{0\} \times(0, \infty)$; so that we set $t_{0}:=0$.

- A function $x_{0} \in C^{2}(\mathbb{R}, \mathbb{R})$ is solution of

$$
\ddot{x}+2 \omega \delta \dot{x}+\omega x=0, \quad x(0)=0, \quad \dot{x}_{0}(0)=\dot{x}_{0}
$$

if and only if 


$$
x_{0}(t)=e^{-\omega \delta t} \frac{\dot{x}_{0}}{\Omega_{\omega}} \sin \Omega_{\omega} t
$$

Moreover,

$$
\dot{x}_{0}(T)=0 \Leftrightarrow \tan \Omega_{\omega} T=\frac{\Omega_{\omega}}{\omega \delta} .
$$

The smallest positive $T$ satisfying (3.28) gives $T_{\omega}$ in (3.23). As a consequence,

$$
x_{0}\left(T_{\omega}\right)=e^{-\omega \delta T_{\omega}} \frac{\dot{x}_{0}}{\Omega_{\omega}} \sin \Omega_{\omega} T_{\omega}
$$

and, since $T_{\omega}$ is minimum, we have $\Omega_{\omega} T_{\omega} \in(0, \pi / 2)$.

- Let $x_{1}$ be the solution of

$$
\ddot{x}+2 \delta \dot{x}+x=0, \quad x(0)=x_{0}\left(T_{\omega}\right), \quad \dot{x}(0)=0 .
$$

If we write $x_{1}$ in the form

$$
x_{1}(t)=e^{-\delta t}\left(A \cos \Omega_{1} t+B \sin \Omega_{1} t\right),
$$

we have

$$
A=x_{0}\left(T_{\omega}\right), \quad \frac{A}{B}=\frac{\Omega_{1}}{\delta} .
$$

Besides,

$$
x_{1}(T)=0 \Leftrightarrow \tan \Omega_{1} T=-\frac{\Omega_{1}}{\delta} .
$$

The smallest positive $T$ satisfying this equation gives $T_{-}$in (3.24). We have also $\Omega_{1} T_{-} \in(\pi / 2, \pi)$. From (3.29) and (3.26), we deduce that

$$
\dot{x}_{1}\left(T_{-}\right)=-k(\omega) \dot{x}_{0}
$$

where

$$
k(\omega):=\exp \left(-\omega \delta T_{\omega}-\delta T_{-}\right) \sin \left(\Omega_{\omega} T_{\omega}\right) \sin \left(\Omega_{1} T_{-}\right) \frac{\delta^{2}+\Omega_{1}^{2}}{\Omega_{1} \Omega_{\omega}} .
$$

Since $\Omega_{\omega} T_{\omega} \in(0, \pi / 2)$ and $\Omega_{1} T_{-} \in(\pi / 2, \pi), k(\omega)$ is positive. Moreover, with (3.28) and (3.30), we infer

$$
\sin ^{2}\left(\Omega_{\omega} T_{\omega}\right)=\frac{\Omega_{\omega}^{2}}{\omega^{2} \delta^{2}+\Omega_{\omega}^{2}}, \quad \sin ^{2}\left(\Omega_{1} T_{-}\right)=\frac{\Omega_{1}^{2}}{\delta^{2}+\Omega_{1}^{2}} .
$$

Thus

$$
k(\omega)^{2}=\exp \left(-2 \omega \delta T_{\omega}-2 \delta T_{-}\right) \frac{1}{\omega},
$$

and (3.26) follows. Since the equation is autonomous, we obtain the existence of a sequence $\left(t_{n}\right)_{n \geq 0}$ satisfying the four properties of the theorem.

Next, we will investigate some properties of $k(\omega)$. 
Proposition 3.7. Let $\delta \in(0,1)$ and $k:\left(0, \frac{1}{\delta^{2}}\right) \rightarrow(0, \infty)$ be the function defined by (3.26). Then $k$ is decreasing on $\left(0,1 / \delta^{2}\right)$ from $\infty$ to $k\left({\frac{1}{\delta^{2}}}^{-}\right)$. Besides, $k\left({\frac{1}{\delta^{2}}}^{-}\right)<\frac{\delta}{e}<1$ and

$$
k(\omega) \simeq \frac{e^{-\delta T_{-}}}{\sqrt{\omega}} \quad \text { when } \omega \rightarrow 0 .
$$

As a consequence, there exists a unique number denoted by $\omega(\delta)$, satisfying

$$
k(\omega(\delta))=1 .
$$

Moreover, $\omega(\delta)<1, \omega\left(0^{+}\right)=1, \omega\left(1^{-}\right)=0$, and, setting $X_{1}:=\left(\frac{1}{\delta^{2}}-1\right)^{1 / 2}$, there holds (see Figure 2)

$$
\exp \left(-\frac{2 \pi}{X_{1}}\right) \leq \omega(\delta) \leq \exp \left(-\frac{2 \pi}{X_{1}}+2 \frac{\arctan X_{1}}{X_{1}}\right)
$$

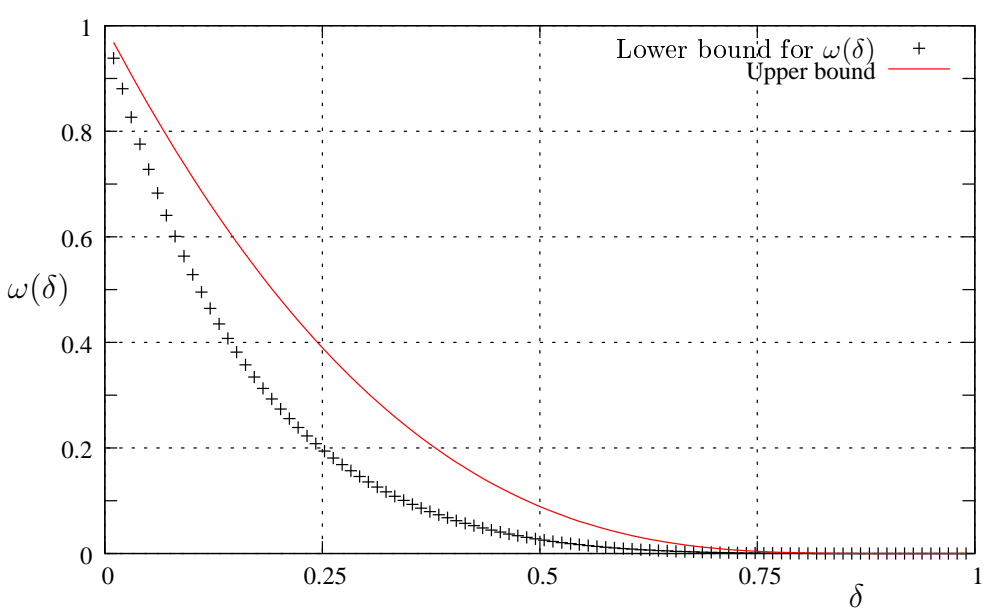

Figure 2. Bounds for $\omega(\delta)$ given by (3.31), where $\omega(\delta)$ is the unique value of $\omega$ for which the homogenous equation (1.1) has a periodic solution.

From (3.31), we deduce the less sharp but simpler estimates for $\omega(\delta)$ :

$$
\exp \left(-\frac{2 \pi}{X_{1}}\right) \leq \omega(\delta) \leq \exp \left(-\frac{\pi}{X_{1}}\right)
$$

Proof. Setting

we obtain with (3.23),

$$
X_{\omega}:=\frac{\Omega_{\omega}}{\omega \delta}=\left(\frac{1}{\omega \delta^{2}}-1\right)^{1 / 2}
$$

$$
\omega \delta T_{\omega}=\frac{1}{X_{\omega}} \arctan X_{\omega} .
$$

By a concavity argument, $y \mapsto \frac{\arctan y}{y}$ is decreasing on $(0, \infty)$. Thus

$$
\omega \mapsto \exp \left(-\omega \delta T_{\omega}\right)
$$


is decreasing on $\left(0, \frac{1}{\delta^{2}}\right)$, and so $k$ does. By (3.33),

$$
\omega \delta T_{\omega} \rightarrow 1 \quad \text { as } \omega \rightarrow \frac{1}{\delta^{2}}
$$

Thus

$$
k\left({\frac{1}{\delta^{2}}}^{-}\right)=\delta \exp \left(-1-\delta T_{-}\right)<\frac{\delta}{e} .
$$

Let us now look at $k(\omega)$ for $\omega$ close to 0 . When $\omega \rightarrow 0, X_{\omega} \rightarrow \infty$. Thus (see (3.33)),

$$
\omega \delta T_{\omega} \rightarrow 0
$$

We conclude with (3.26).

Let us now consider the solution $\omega(\delta)$ to $k(\omega)=1$. By (3.26), (3.24),

$$
\omega(\delta) \leq \exp \left(-2 \delta T_{-}\right)=\exp \left(-\frac{2 \pi}{X_{1}}+2 \frac{\arctan X_{1}}{X_{1}}\right) .
$$

Regarding the lower bound, since $k$ decreases and $k(1)<1$, we have $\omega(\delta)<1$. Hence,

$$
\omega(\delta) \delta T_{\omega(\delta)}=\frac{1}{X_{\omega(\delta)}} \arctan X_{\omega(\delta)} \leq \frac{1}{X_{1}} \arctan X_{1} .
$$

Thus, with (3.24),

$$
\sqrt{\omega(\delta)} \geq \exp \left(-\frac{1}{X_{1}} \arctan X_{1}-\delta T_{-}\right)=\exp \left(-\frac{\pi}{X_{1}}\right) .
$$

This proves (3.31).

Finally, $\omega\left(0^{+}\right)=1$ and $\omega\left(1^{-}\right)=0$ follow from $(3.32)$.

\section{Asymptotic properties of the solutions to (1.1)}

We start with the homogenous case. In this situation, the long time behavior of the system is completely known and depends on the value of $k(\omega)$. More precisely, we have the following statement.

Corollary 4.1. Under the assumptions and notation of Propositions 3.6 and 3.7, if

- $k(\omega)<1$ i.e. $\omega>\omega(\delta)$ then $|x(t)|+|\dot{x}(t)| \rightarrow 0$ as $t \rightarrow \infty$;

- $k(\omega)=1$ i.e. $\omega=\omega(\delta)$ then $x$ is a $2\left(T_{\omega}+T_{-}\right)$- periodic fonction;

- $k(\omega)>1$ i.e. $\omega<\omega(\delta)$ then $|x(t)|+|\dot{x}(t)| \rightarrow \infty$ as $t \rightarrow \infty$.

Remark 4.1. The case $k(\omega)=1$ is surprising since from the point of view of application it implies that power can be extracted without wave! By Proposition 3.7, for any admissible $\delta$, we can choose appropriate $\omega$ so that $k(\omega)=1$. This suggests that the model (1.1) should be modified in order to avoid such periodic solutions.

There results from the proof below that the decay (respectively, growth) rate toward zero (infinity) is exponential. 
Proof. According to Proposition 3.6 and (3.29), for all $n \geq 0$, we have

$$
\begin{array}{ll}
x\left(t_{4 n}\right)=0 & \dot{x}\left(t_{4 n}\right)=k(\omega)^{2 n} \dot{x}\left(t_{0}\right) \\
x\left(t_{4 n+1}\right)=e^{-\omega \delta T_{\omega}} \frac{\sin \left(\Omega_{\omega} T_{\omega}\right)}{\Omega_{\omega}} \dot{x}\left(t_{4 n}\right) & \dot{x}\left(t_{4 n+1}\right)=0 \\
x\left(t_{4 n+2}\right)=0 & \dot{x}\left(t_{4 n+2}\right)=-k(\omega) \dot{x}\left(t_{4 n}\right) \\
x\left(t_{4 n+3}\right)=e^{-\omega \delta T_{\omega}} \frac{\sin \left(\Omega_{\omega} T_{\omega}\right)}{\Omega_{\omega}} \dot{x}\left(t_{4 n+2}\right) & \dot{x}\left(t_{4 n+3}\right)=0 .
\end{array}
$$

- If $k(\omega)<1$ then the above equalities imply

$$
\left|x\left(t_{n}\right)\right|+\left|\dot{x}\left(t_{n}\right)\right| \underset{n \rightarrow \infty}{\longrightarrow} 0 .
$$

Let $n \in \mathbb{N}$ and $t \in\left(t_{2 n}, t_{2 n+1}\right)$. By (2) Proposition 3.6, $V(t):=(x(t), \dot{x}(t), t)^{t} \in A_{+}$. Thus, if we multiply the first equation of (1.1) by $\dot{x}$ and integrate between $t_{2 n}$ and $t$, we get

$$
\left(\frac{1}{2} \dot{x}^{2}+\frac{\omega}{2} x^{2}\right)(t)+2 \omega \delta \int_{t_{2 n}}^{t} \dot{x}^{2}(s) \mathrm{d} s=\left(\frac{1}{2} \dot{x}^{2}+\frac{\omega}{2} x^{2}\right)\left(t_{2 n}\right) .
$$

In a same way, if $t \in\left(t_{2 n+1}, t_{2 n+2}\right)$ then $V(t) \in A_{-}$and

$$
\left(\frac{1}{2} \dot{x}^{2}+\frac{1}{2} x^{2}\right)(t)+2 \delta \int_{t_{2 n+1}}^{t} \dot{x}^{2}(s) \mathrm{d} s=\left(\frac{1}{2} \dot{x}^{2}+\frac{1}{2} x^{2}\right)\left(t_{2 n+1}\right) .
$$

Combining (4.1)-(4.3), we obtain $|x(t)|+|\dot{x}(t)| \rightarrow 0$.

- If $k(\omega)>1$ then, since $\dot{x}\left(t_{0}\right) \neq 0$ according to Proposition 3.6, we have

$$
\left|x\left(t_{n}\right)\right|+\left|\dot{x}\left(t_{n}\right)\right| \underset{n \rightarrow \infty}{\longrightarrow} \infty .
$$

For all $t \in\left(t_{2 n}, t_{2 n+1}\right)$, there holds (see (4.2))

$$
\left(\frac{1}{2} \dot{x}^{2}+\frac{\omega}{2} x^{2}\right)\left(t_{2 n+1}\right)+2 \omega \delta \int_{t}^{t_{2 n+1}} \dot{x}^{2}(s) \mathrm{d} s=\left(\frac{1}{2} \dot{x}^{2}+\frac{\omega}{2} x^{2}\right)(t) .
$$

Hence,

$$
\lim _{t \rightarrow \infty, t \in \cup_{n \geq 0}\left[t_{2 n}, t_{2 n+1}\right]}|x(t)|+|\dot{x}(t)|=\infty .
$$

Arguing in a same way on $A_{-}$, we obtain the convergence toward $\infty$.

- If $k(\omega)=1$ then

$$
\left(x\left(t_{4}\right), \dot{x}\left(t_{4}\right)\right)=\left(x\left(t_{0}\right), \dot{x}\left(t_{0}\right)\right) .
$$

Since the equation is autonomous and Theorem 3.4 provides an uniqueness result, $x$ is periodic.

In the sequel, we give a bounded-input-bounded-output stability result. The main tools are a rescaling argument and an energy method. Fairly general forcing terms $g$ are allowed. In particular, $g$ is not assumed to be periodic. 
Theorem 4.2. Under assumption (2.1), let us suppose, in addition that

$$
\begin{aligned}
& \omega \delta^{2}>1 / 2 \\
& \delta>1 / \sqrt{2} \\
& g \quad \text { is bounded on }[0, \infty) .
\end{aligned}
$$

Then Equation (1.1) admits a bounded absorbing set in the phase space $\mathbb{R}^{2}$. In particular, for all positive $\varepsilon$ and any global solution $x$ of $(1.1)$ on $[0, \infty)$, there exists a time $T>0$ (depending on $\varepsilon, x(0)$ and $\dot{x}(0)$ ) such that for $t>T$,

$$
\begin{aligned}
& |x(t)| \leq \sqrt{8 \delta^{3} C(\delta, \omega)}\|g\|_{\infty}+\varepsilon \\
& |\dot{x}(t)| \leq 2 \sqrt{\delta C(\delta, \omega)}\|g\|_{\infty}+\varepsilon
\end{aligned}
$$

where

$$
\begin{aligned}
\|g\|_{\infty} & :=\sup _{t \geq 0}|g(t)| \\
C(\delta, \omega) & :=\frac{\delta}{4} \max \left(\frac{\omega^{2}}{2 \omega \delta^{2}-1}, \frac{1}{2 \delta^{2}-1}\right) .
\end{aligned}
$$

Proof. Let $x$ be a global solution of (1.1) in the sense of Definition 2.2. Without loss of generality, we may assume that, for some positive $\varepsilon$,

$$
V((0, \varepsilon)) \subset A_{+} \cup A_{0++} .
$$

Next, we claim that for all $T>0$, there exists a nonnegative integer $N$ and a sequence $0=t_{0}<t_{1}<\cdots<t_{N}<t_{N+1}:=T$ such that for $n \geq 0$,

$$
\begin{array}{ll}
\ddot{x}+2 \omega \delta \dot{x}+\omega x=\omega g & \text { on }\left(t_{2 n}, t_{2 n+1}\right) \\
\ddot{x}+2 \delta \dot{x}+x=g & \text { on }\left(t_{2 n+1}, t_{2 n+2}\right) .
\end{array}
$$

Since the assumptions on $g$ are weaker than the ones in Theorem 3.1, this claim has to be justified. If $V((0, T)) \subset A_{+} \cup A_{0++}$ then we put $N:=0, t_{1}:=T$ so that, by Definition 2.1, (4.10) holds on $(0, T)=\left(t_{0}, t_{1}\right)$. Otherwise, $V$ leaves $A_{+} \cup A_{0++}$ at some time $t_{1}>0$. Thus since $x$ is a local solution, there exists $\varepsilon>0$ such that

$$
V\left(\left(t_{1}, t_{1}+\varepsilon\right)\right) \subset A_{-}
$$

and $x$ solves $(4.11)$ on $\left(t_{1}, t_{1}+\varepsilon\right)$. Then the claim follows easily by induction.

Next we set

$$
\begin{aligned}
\beta & :=\frac{1}{2 \delta} \\
y(t) & :=e^{\beta t} x(t) \\
E(t) & :=\frac{1}{2} \dot{y}^{2}(t)+\frac{1}{8 \delta^{2}} y^{2}(t) \quad \forall t \geq 0 .
\end{aligned}
$$

Then for $t \in\left(0, t_{1}\right)$,

$$
\ddot{y}+2(\omega \delta-\beta) \dot{y}+\frac{1}{4 \delta^{2}} y=e^{\beta t} \omega g(t)
$$


and

$$
E(t)+2(\omega \delta-\beta) \int_{0}^{t} \dot{y}^{2}(s) \mathrm{d} s=\int_{0}^{t} e^{\beta s} \omega g(s) \dot{y}(s) \mathrm{d} s+E(0) .
$$

Due to (4.5),

$$
\omega \delta-\beta=\omega \delta-\frac{1}{2 \delta}>0 .
$$

So, using Young's inequality

$$
a b \leq 2(\omega \delta-\beta) a^{2}+\frac{1}{8(\omega \delta-\beta)} b^{2},
$$

we derive from (4.13)

$$
E(t) \leq \frac{\omega^{2}}{8(\omega \delta-\beta)} \int_{0}^{t} e^{2 \beta s} g^{2}(s) \mathrm{d} s+E(0) .
$$

In particular, since $E$ is continuous on $\mathbb{R}$,

$$
E\left(t_{1}\right) \leq \frac{\omega^{2}}{8(\omega \delta-\beta)} \int_{0}^{t_{1}} e^{2 \beta s} g^{2}(s) \mathrm{d} s+E(0) .
$$

If $N \geq 1$ then, noticing that $E$ is independent of $\omega$ - this fact is the key point of the proof - we have

$$
E\left(t_{2}\right) \leq \frac{1}{8(\delta-\beta)} \int_{t_{1}}^{t_{2}} e^{2 \beta s} g^{2}(s) \mathrm{d} s+E\left(t_{1}\right)
$$

Hence, (4.15), (4.16) and the notation (4.9) imply

$$
E\left(t_{2}\right) \leq C(\delta, \omega) \int_{0}^{t_{2}} e^{2 \beta s} g^{2}(s) \mathrm{d} s+E(0) .
$$

By induction, there results

$$
E(T) \leq C(\delta, \omega) \frac{e^{2 \beta T}}{2 \beta}\|g\|_{\infty}^{2}+E(0) .
$$

Let us go back to the function $x$. For all $t \geq 0$, we have

$$
2 E(t)=e^{2 \beta t}\left((\dot{x}+\beta x)^{2}+\beta^{2} x^{2}\right)(t) .
$$

Thus

$$
(\dot{x}+\beta x)^{2}(t)+\beta^{2} x^{2}(t) \leq \frac{C(\delta, \omega)\|g\|_{\infty}^{2}}{\beta}+2 e^{-2 \beta t} E(0) .
$$

Using

we derive

$$
\frac{1}{2} a^{2} \leq(a+b)^{2}+b^{2}
$$

$$
\begin{aligned}
& \dot{x}(t)^{2} \leq 4 \delta C(\delta, \omega)\|g\|_{\infty}^{2}+4 e^{-\frac{1}{\delta} t} E(0) \\
& x(t)^{2} \leq 8 \delta^{3} C(\delta, \omega)\|g\|_{\infty}^{2}+8 \delta^{2} e^{-\frac{1}{\delta} t} E(0) .
\end{aligned}
$$

This completes the proof of the theorem. 
Remark 4.2. An immediate consequence of Theorem 4.2 is that any periodic solution $x$ and a fortiori, any limit cycle of (1.1) satisfy

$$
\begin{aligned}
& |x(t)| \leq \sqrt{8 \delta^{3} C(\delta, \omega)}\|g\|_{\infty} \\
& |\dot{x}(t)| \leq 2 \sqrt{\delta C(\delta, \omega)}\|g\|_{\infty}, \quad \forall t \in \mathbb{R} .
\end{aligned}
$$

\section{Periodic Solutions}

If $\omega=1$ and $g$ is periodic then (1.1) reduces to a linear equation which admits a periodic solution. Hence we will use a perturbation argument to prove that, for $\omega \simeq 1$, (1.1) posseses periodic solutions. This argument is based on the implicit function theorem. However, due to the discontinuity of $\ddot{x}$, standard results on the persistence of periodic solutions do not apply here. Accordingly, we will use the fact that explicit solutions are available for suitable forcing $g$. We then perform a perturbation argument on the coefficients of these explicit solutions. We get an algebraic equation in $\mathbb{R}^{12}$ parametrized by $\omega$.

Theorem 5.1. Let us assume that

$$
\begin{aligned}
& \delta \in(0,1), \quad \omega \delta^{2} \in(0,1) \\
& g(t)=g_{0} \sin (\alpha t) \quad \forall t \in \mathbb{R}
\end{aligned}
$$

where

$$
\alpha>0, \quad g_{0} \in \mathbb{R}^{*} .
$$

Then for $\omega$ close enough to 1 , (1.1) admits a $\frac{2 \pi}{\alpha}$-periodic solution.

Proof. Without loss of generality, we may assume $g_{0}=1$. For $\omega=1$, (1.1) becomes

$$
\ddot{x}+2 \delta \dot{x}+x=\sin (\alpha t) .
$$

Let the function $x_{p, 1}$ be defined by

$$
x_{p, 1}(t)=X_{1} \sin \left(\alpha t-\theta_{1}\right), \quad \forall t \in \mathbb{R},
$$

with

$$
X_{1}=\frac{1}{\left[\left(1-\alpha^{2}\right)^{2}+4 \delta^{2} \alpha^{2}\right]^{1 / 2}}, \quad \theta_{1}=\left\{\begin{array}{ll}
\arctan \frac{2 \delta \alpha}{1-\alpha^{2}} & \text { if } 0<\alpha<1 \\
\frac{\pi}{2} & \text { if } \alpha=1 \\
\pi+\arctan \frac{2 \delta \alpha}{1-\alpha^{2}} & \text { if } 1<\alpha
\end{array} .\right.
$$

Remark that $\theta_{1}$ belongs to $(0, \pi)$ and that $x_{p, 1}$ is a $\frac{2 \pi}{\alpha}$-periodic solution to $(5.4)$. Moreover, if we set

$$
\begin{aligned}
& t_{k}:=\frac{\theta_{1}}{\alpha}+\frac{k \pi}{2 \alpha}, \quad \forall k=0,1,2,3,4 \\
& V(t):=\left(x_{p, 1}(t), \dot{x}_{p, 1}(t), t\right)^{t}
\end{aligned}
$$

then for $i=0,1$,

$$
V\left(\left(t_{2 i}, t_{2 i+1}\right)\right) \subset A_{+}, \quad V\left(\left(t_{2 i+1}, t_{2 i+2}\right)\right) \subset A_{-} .
$$


For $\omega \simeq 1$, we define $x_{p, \omega}$ by

$$
x_{p, \omega}(t)=X_{\omega} \sin \left(\alpha t-\theta_{\omega}\right), \quad \forall t \in \mathbb{R},
$$

with

$$
X_{\omega}=\frac{\omega}{\left[\left(\omega-\alpha^{2}\right)^{2}+4 \omega^{2} \delta^{2} \alpha^{2}\right]^{1 / 2}}, \quad \theta_{\omega}= \begin{cases}\arctan \frac{2 \omega \delta \alpha}{\omega-\alpha^{2}} & \text { if } \alpha^{2}<\omega \\ \frac{\pi}{2} & \text { if } \omega=\alpha^{2} . \\ \pi+\arctan \frac{2 \omega \delta \alpha}{\omega-\alpha^{2}} & \text { if } \omega<\alpha^{2}\end{cases}
$$

Then, for $\omega \simeq 1$, a function $x \in C^{1}(\mathbb{R}, \mathbb{R})$ is a $\frac{2 \pi}{\alpha}$-periodic solution to (1.1) on $\mathbb{R}$ in the sense of Definition 2.2, if there exist 12 real numbers $A_{0}, B_{0}, \ldots, A_{3}, B_{3}$ and $t_{0}(\omega)<t_{1}(\omega)<t_{2}(\omega)<t_{3}(\omega)$ such that the four following conditions hold true.

- For all $t \in\left(t_{0}(\omega), t_{1}(\omega)\right)$,

$$
x(t)=x_{0}(t):=e^{-\omega \delta t}\left(A_{0} \cos \Omega_{\omega} t+B_{0} \sin \Omega_{\omega} t\right)+x_{p, \omega}(t)
$$

(recall that $\Omega_{\omega}:=\sqrt{\omega}\left(1-\omega \delta^{2}\right)^{1 / 2}$, see $\left.(3.22)\right)$ and

$$
\begin{aligned}
& x_{0}\left(t_{0}(\omega)\right)=0, \quad \dot{x}_{0}\left(t_{1}(\omega)\right)=0 \\
& V\left(\left(t_{0}(\omega), t_{1}(\omega)\right)\right) \subset A_{+} .
\end{aligned}
$$

- For all $t \in\left(t_{1}(\omega), t_{2}(\omega)\right)$,

$$
x(t)=x_{1}(t):=e^{-\delta t}\left(A_{1} \cos \Omega_{1} t+B_{1} \sin \Omega_{1} t\right)+x_{p, 1}(t)
$$

and

$$
\begin{aligned}
& x_{1}\left(t_{1}(\omega)\right)=x_{0}\left(t_{1}(\omega)\right), \quad \dot{x}_{1}\left(t_{1}(\omega)\right)=0, \quad x_{1}\left(t_{2}(\omega)\right)=0 \\
& V\left(\left(t_{1}(\omega), t_{2}(\omega)\right)\right) \subset A_{-} .
\end{aligned}
$$

- For all $t \in\left(t_{2}(\omega), t_{3}(\omega)\right)$,

$$
x(t)=x_{2}(t):=e^{-\omega \delta t}\left(A_{2} \cos \Omega_{\omega} t+B_{2} \sin \Omega_{\omega} t\right)+x_{p, \omega}(t)
$$

and

$$
\begin{aligned}
& \dot{x}_{2}\left(t_{2}(\omega)\right)=\dot{x}_{1}\left(t_{2}(\omega)\right), \quad x_{2}\left(t_{2}(\omega)\right)=0, \quad \dot{x}_{2}\left(t_{3}(\omega)\right)=0 \\
& V\left(\left(t_{2}(\omega), t_{3}(\omega)\right)\right) \subset A_{+} .
\end{aligned}
$$

- For all $t \in\left(t_{3}(\omega), t_{4}(\omega)\right)$ where $t_{4}(\omega):=t_{0}(\omega)+\frac{2 \pi}{\alpha}$,

$$
x(t)=x_{3}(t):=e^{-\delta t}\left(A_{3} \cos \Omega_{1} t+B_{3} \sin \Omega_{1} t\right)+x_{p, 1}(t)
$$

and

$$
\begin{aligned}
& x_{3}\left(t_{3}(\omega)\right)=x_{2}\left(t_{3}(\omega)\right), \dot{x}_{3}\left(t_{3}(\omega)\right)=0, x_{3}\left(t_{4}(\omega)\right)=0, \dot{x}_{3}\left(t_{4}(\omega)\right)=\dot{x}_{0}\left(t_{0}(\omega)\right) \\
& V\left(\left(t_{3}(\omega), t_{4}(\omega)\right)\right) \subset A_{-} .
\end{aligned}
$$

Since $t_{4}(\omega)=t_{0}(\omega)+\frac{2 \pi}{\alpha},(5.6)-(5.9)$ contain 12 scalar equations with 12 unknowns $A_{0}, \ldots, B_{3}, t_{0}(\omega), \ldots, t_{3}(\omega)$ and one parameter, namely $\omega$. 
For $\omega=1, x_{p, 1}$ gives a solution to these equations. Indeed,

$$
\begin{aligned}
& A_{0}=\cdots=B_{3}=0 \\
& t_{k}(1):=t_{k}=\frac{\theta_{1}}{\alpha}+\frac{k \pi}{2 \alpha}, \quad \forall k=0,1,2,3 .
\end{aligned}
$$

solve (5.6)-(5.9) for $\omega=1$.

Next, we use the implicit function theorem to show the persistence of the solution to (5.6)-(5.9). For this, we have to compute the jacobian matrix of the underlying function at the point given by (5.10) and for $\omega=1$. Afterwards, we will prove that this matrix is invertible.

Its first line is computed by differentiating the first equation of (5.6), namely $x_{0}\left(t_{0}(\omega)\right)=0$. For simplicity, we define

$$
u(t):=e^{-\delta t} \cos \Omega_{1} t, \quad v(t):=e^{-\delta t} \sin \Omega_{1} t, \quad \forall t \in \mathbb{R} .
$$

Then we get

$$
\left.\frac{\partial}{\partial A_{0}} x_{0}\left(t_{0}(\omega)\right)\right|_{\left(A_{0}, \ldots, B_{3}, t_{0}(\omega), \ldots, t_{3}(\omega), \omega\right)=\left(0, \ldots, 0, t_{0}, \ldots, t_{3}, 1\right)}=u\left(t_{0}\right)=u\left(\frac{\theta_{1}}{\alpha}\right) .
$$

At the same point, we have also

$$
\frac{\partial}{\partial B_{0}} x_{0}\left(t_{0}(\omega)\right)=v\left(t_{0}\right), \quad \frac{\partial}{\partial t_{0}} x_{0}\left(t_{0}(\omega)\right)=\dot{x}_{p, 1}\left(t_{0}\right)=\alpha X_{1} .
$$

The derivative with respect to the other variables vanish. Hence there is only 3 non trivial entries in the first line of the jacobian matrix. Taking into account the other equations in (5.6)-(5.9), the jacobian matrix corresponding to (5.6)-(5.9) reads

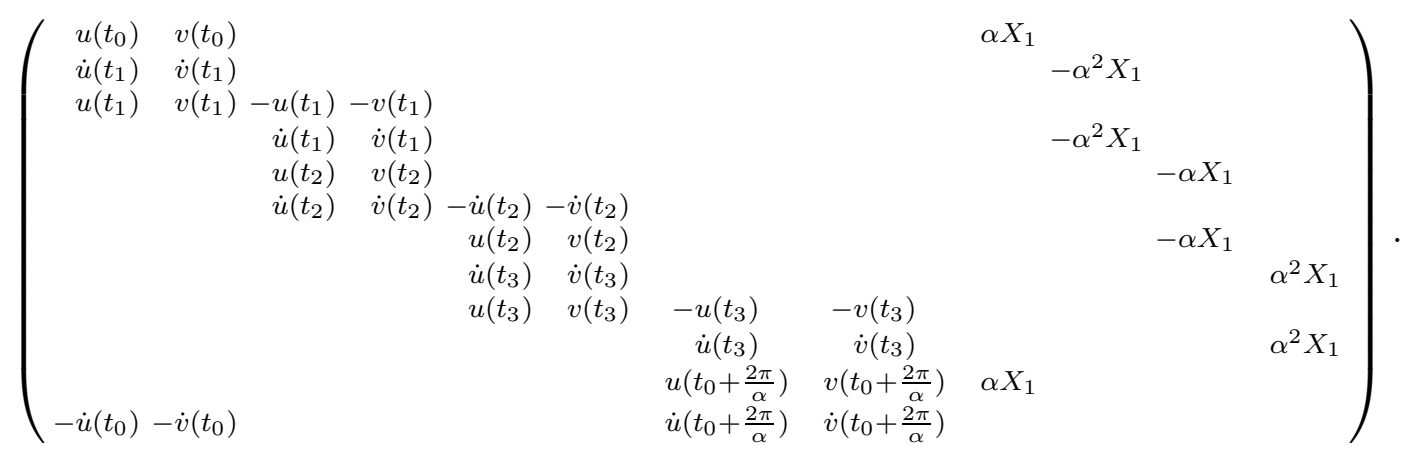

In the above matrix, the first column contains the partial derivative with respect to $A_{0}$. The $8^{\text {th }}$ and $9^{\text {th }}$ columns contain the partial derivative with respect to $B_{3}$ and $t_{0}$. Zero entries are omitted.

Now, we will apply elementary transformations to this matrix what will do not change its invertibility. First, we replace the

- $11^{\text {th }}$ line by the difference of the $11^{\text {th }}$ line and the $1^{\text {st }}$ line;

- $8^{\text {th }}$ line by the difference of the $8^{\text {th }}$ line and the $10^{\text {th }}$ line;

- $5^{\text {th }}$ line by the difference of the $5^{\text {th }}$ line and the $7^{\text {th }}$ line;

- $2^{\text {nd }}$ line by the difference of the $2^{\text {nd }}$ line and the $4^{\text {th }}$ line. 
Thus the 4 last columns contain only one non zero entry. This leads us to the following $8 \times 8$ matrix.

$$
\left(\begin{array}{rrrrrrrr}
\dot{u}\left(t_{1}\right) & \dot{v}\left(t_{1}\right) & -\dot{u}\left(t_{1}\right) & -\dot{v}\left(t_{1}\right) & & & & \\
u\left(t_{1}\right) & v\left(t_{1}\right) & -u\left(t_{1}\right) & -v\left(t_{1}\right) & & & & \\
& & u\left(t_{2}\right) & v\left(t_{2}\right) & -u\left(t_{2}\right) & -v\left(t_{2}\right) & & \\
& & \dot{u}\left(t_{2}\right) & \dot{v}\left(t_{2}\right) & -\dot{u}\left(t_{2}\right) & -\dot{v}\left(t_{2}\right) & & \\
& & & & \dot{u}\left(t_{3}\right) & \dot{v}\left(t_{3}\right) & -\dot{u}\left(t_{3}\right) & -\dot{v}\left(t_{3}\right) \\
-u\left(t_{0}\right) & -v\left(t_{0}\right) & & & u\left(t_{3}\right) & v\left(t_{3}\right) & -u\left(t_{3}\right) & -v\left(t_{3}\right) \\
-\dot{u}\left(t_{0}\right) & -\dot{v}\left(t_{0}\right) & & & & & u\left(t_{4}\right) & v\left(t_{4}\right) \\
& & & & & \dot{u}\left(t_{4}\right) & \dot{v}\left(t_{4}\right)
\end{array}\right)
$$

We write this matrix under the form

$$
\left(\begin{array}{rrrr}
A & -A & & \\
& B & -B & \\
& & C & -C \\
D & & & E
\end{array}\right)
$$

where $A, \ldots, E$ are $2 \times 2$ matrices defined in an obvious way according to (5.11). Remark that $A, \ldots, E$ are invertible since their determinant is (up to a sign) a wronskien of $u$ and $v$.

In the above matrix, we replace the

- $2^{\text {nd }}$ column by the sum of the $2^{\text {nd }}$ and the $1^{\text {st }}$ column;

- $3^{\text {rd }}$ column by the sum of the $3^{\text {rd }}$ and the $2^{\text {nd }}$ column;

- $4^{\text {th }}$ column by the sum of the $4^{\text {th }}$ and the $3^{\text {rd }}$ column.

We get

$$
\left(\begin{array}{llll}
A & & & \\
& B & & \\
& & C & \\
D & D & D & D+E
\end{array}\right)
$$

By multiplying this matrix to the right by the invertible matrix

$$
\left(\begin{array}{cccc}
I & & & \\
& I & I & \\
-D A^{-1} & -D B^{-1} & -D C^{-1} & I
\end{array}\right)
$$

(where $I$ denotes the identity matrix of $\mathbb{R}^{2}$ ), we get

$$
\left(\begin{array}{llll}
A & & & \\
& B & & \\
& & C & \\
& & & D+E
\end{array}\right) .
$$

Since, as explain above, $A, \ldots, E$ are invertible, there remains to compute the determinant of $D+E$. Setting $T:=\frac{2 \pi}{\alpha}$, this determinant is the wronskien of $u(\cdot+T)-u$ 
and $v(\cdot+T)-v$ at time $t_{0}$. Thus it is enough to compute this wronskien for $t=0$. This gives

$$
\Omega_{1}\left(e^{-2 \delta T}+1-2 e^{-\delta T} \cos \Omega_{1} T\right) .
$$

Thus, if the determinant of $D+E$ vanishes then $\cos \Omega_{1} T=\operatorname{ch} \delta \mathrm{T}$ and $\delta T=0$. Our assumptions on $\delta$ imply that $\operatorname{det}(D+E) \neq 0$. Then, for $\omega \simeq 1,(5.6)-(5.9)$ has a solution that we denote by

$$
A_{0}(\omega), \ldots, B_{0}(\omega), t_{0}(\omega), \ldots, t_{3}(\omega) .
$$

By choosing $\omega$ close enough to 1 , we have in addition

$$
t_{0}(\omega)<t_{1}(\omega)<t_{2}(\omega)<t_{3}(\omega) .
$$

Then we define $x: \mathbb{R} \rightarrow \mathbb{R}$ in the following way. For $k=0,1$,

$x(t):= \begin{cases}e^{-\omega \delta t}\left(A_{2 k}(\omega) \cos \Omega_{\omega} t+B_{2 k}(\omega) \sin \Omega_{\omega} t\right)+x_{p, \omega}(t) & \text { if } t \in\left[t_{2 k}(\omega), t_{2 k+1}(\omega)\right) \\ e^{-\delta t}\left(A_{2 k+1}(\omega) \cos \Omega_{1} t+B_{2 k+1}(\omega) \sin \Omega_{1} t\right)+x_{p, 1}(t) & \text { if } t \in\left[t_{2 k+1}(\omega), t_{2 k+2}(\omega)\right) .\end{cases}$

Outside of $\left[t_{0}(\omega), t_{4}(\omega)\right)$, we extend $x$ by periodicity. From (5.6)-(5.9), there results that $x$ is a $C^{1}$ function and is $T$-periodic. Thus there remains to check that $x \dot{x}$ is positive on $\left(t_{2 k}(\omega), t_{2 k+1}(\omega)\right)$ and negative on $\left(t_{2 k+1}(\omega), t_{2 k+2}(\omega)\right)$. We will only prove that $x \dot{x}$ is positive on $\left(t_{0}(\omega), t_{1}(\omega)\right)$. For this, we set

$$
y(t, \omega):=x_{0}\left(t+t_{0}(\omega)\right) \dot{x}_{0}\left(t+t_{0}(\omega)\right), \quad \forall t \in \mathbb{R}
$$

where

$$
x_{0}(t):=e^{-\omega \delta t}\left(A_{0}(\omega) \cos \Omega_{\omega} t+B_{0}(\omega) \sin \Omega_{\omega} t\right)+x_{p, \omega}(t) .
$$

Since $\dot{y}(0,1)=\alpha^{2} X_{1}^{2}$, and $y$ is of class $C^{2}$, there exists $\varepsilon \in(0,1)$ such that for all $\omega \in(1-\varepsilon, 1+\varepsilon)$,

$$
\begin{aligned}
& \dot{y}(0, \omega) \geq \frac{1}{2} \dot{y}(0,1) \\
& M:=\sup \left\{|\ddot{y}(t, \omega)| \mid \omega \in(1-\varepsilon, 1+\varepsilon), t \in\left(0, t_{1}(\omega)-t_{0}(\omega)\right\}<\infty .\right.
\end{aligned}
$$

Thus for all $\omega \in(1-\varepsilon, 1+\varepsilon)$,

$$
\begin{aligned}
y(t, \omega) & =t \dot{y}(0, \omega)+t^{2} \int_{0}^{1}(1-s) \ddot{y}(t s, \omega) \mathrm{d} s \\
& \geq \frac{t}{2} \dot{y}(0,1)-\frac{t^{2}}{2} M \\
& \geq \frac{1}{4} \dot{y}(0,1) t
\end{aligned}
$$

provided that $t<\frac{\dot{y}(0,1)}{2 M}$. Arguing in a same way in a neighborhood of $t_{1}(\omega)-t_{0}(\omega)$, we obtain that, for some $\tau>0$ independent of $\omega$,

$$
y(\cdot, \omega)>0 \quad \text { on }(0, \tau] \cup\left[t_{1}(\omega)-t_{0}(\omega)-\tau, t_{1}(\omega)-t_{0}(\omega)\right) .
$$

Since $\tau$ is independent of $\omega$, we may reduce $\varepsilon$ if necessary, so that

$$
t_{1}(\omega)-t_{0}(\omega)-\tau \leq t_{1}-t_{0}-\tau / 2 .
$$


Now

$$
y(\cdot, \omega) \underset{\omega \rightarrow 1}{\longrightarrow} y(\cdot, 1)
$$

uniformly on compact sets and $y(\cdot, 1)$ is positive on $\left(0, t_{1}-t_{0}\right)$, thus for $\omega$ close enough to 1 ,

$$
y(\cdot, \omega)>0 \quad \text { on }\left[\tau, t_{1}-t_{0}-\tau / 2\right] .
$$

Combining (5.14)-(5.16), we derive

$$
y(\cdot, \omega)>0 \quad \text { on }\left(0, t_{1}(\omega)-t_{0}(\omega)\right)
$$

i.e.

$$
x \dot{x}>0 \quad \text { on }\left(t_{0}(\omega), t_{1}(\omega)\right) .
$$

This completes the proof of the theorem.

\section{ENERGy BALANCE}

If there is no mass modulation i.e. $\omega=1$, then the energy balance for Equation (1.1) takes the form

$$
E_{W}+E_{D}=E_{g}
$$

where $E_{W}$ is the energy of the WEC, namely

$$
E_{W}(t)=\frac{1}{2}\left(\dot{x}^{2}+x^{2}\right)(t) .
$$

The damping energy, $E_{D}$ is the sum of the energy harvested by the power takeoff system and the energy dissipated by hydrodynamic damping. According to [OOS10],

$$
\frac{\mathrm{d}}{\mathrm{d} t} E_{D}(t)=2 \delta \dot{x}^{2}(t)
$$

Finally, $E_{g}$, the energy brought by ocean waves has the density

$$
\frac{\mathrm{d}}{\mathrm{d} t} E_{g}(t)=g \dot{x}^{2}(t)
$$

In the case of mass modulation (i.e. $\omega \neq 1$ ), we rewrite $(1.1)$ under the form

$$
\begin{array}{ll}
\frac{1}{\omega} \ddot{x}+2 \delta \dot{x}+x=g & \text { if } x \dot{x}>0 \\
\ddot{x}+2 \delta \dot{x}+x=g & \text { if } x \dot{x}<0 .
\end{array}
$$

Now, we propose the energy balance

$$
E_{W}+E_{D}=E_{g}+E_{m}
$$

where the extra term $E_{m}$ is the energy generated by mass modulation. By (6.4), $E_{g}$ and $E_{D}$ still satisfy (6.2) and (6.3). However, roughly speaking,

$$
E_{W}(t)= \begin{cases}E_{\omega}(t):=\frac{1}{2 \omega} \dot{x}^{2}(t)+\frac{1}{2} x^{2}(t) & \text { if } x \dot{x}>0 \\ E_{1}(t):=\frac{1}{2} \dot{x}^{2}(t)+\frac{1}{2} x^{2}(t) & \text { if } x \dot{x}<0 .\end{cases}
$$


More precisely, assuming (2.1), (2.4), if $x$ denotes a global solution to (1.1) on $\mathbb{R}$, we set for all $t \in \mathbb{R}$ (see Section 2 for the notation),

$$
E_{W}(t):= \begin{cases}E_{\omega}(t) & \text { if } V(t) \in A_{+} \cup A_{0++} \\ E_{1}(t) & \text { if } V(t) \in A_{-} \cup A_{0--}\end{cases}
$$

If $V(t) \notin A_{+} \cup A_{0++} \cup A_{-} \cup A_{0--}$, then by Definition 2.1, there exists $\varepsilon>0$ such that

$$
V((t, t+\varepsilon)) \subset A_{-} \quad \text { or } \quad V((t, t+\varepsilon)) \subset A_{+} .
$$

Hence, we may extend $E_{W}$ by setting

$$
E_{W}(t):=\lim _{s \rightarrow t, s>t} E_{W}(s) .
$$

We will compute the variation of energy during one cycle, where a cycle is illustrated by Figures 1, 3, 4 and 5 .

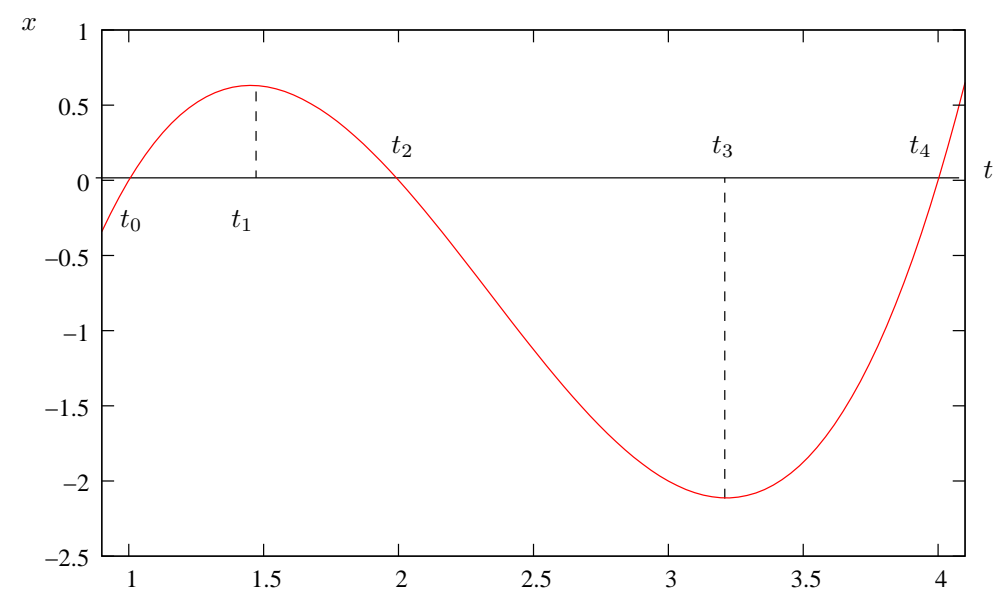

Figure 3. The graph of $t \mapsto x(t)$ corresponding to the cycle in Fig. 1.

The formal definition of a cycle is the following.

Definition 6.1. Assume (2.1), (2.4) and that $x$ is a solution to (1.1) on $\mathbb{R}$ in the sense of Definition 2.2.

- A list $\left(t_{0}, \ldots, t_{4}\right)$ of 5 real numbers is a positive cycle if $t_{0}<\cdots<t_{4}$ and there exists $\varepsilon>0$ such that for $k=0,1$,

$$
\begin{aligned}
& V\left(\left(t_{2 k}, t_{2 k+1}\right)\right) \subset A_{+} \cup A_{0++} \\
& V\left(\left(t_{2 k+1}, t_{2 k+2}\right)\right) \subset A_{-} \cup A_{0--} \\
& V\left(\left(t_{0}-\varepsilon, t_{0}\right)\right) \subset A_{-}, \quad V\left(\left(t_{4}, t_{4}+\varepsilon\right)\right) \subset A_{+} .
\end{aligned}
$$

- The definition of a negative cycle is obtained by exchanging the signs + and - in the definition of a positive cycle.

- $\left(t_{0}, \ldots, t_{4}\right)$ is a cycle if it is a negative or a positive cycle. 


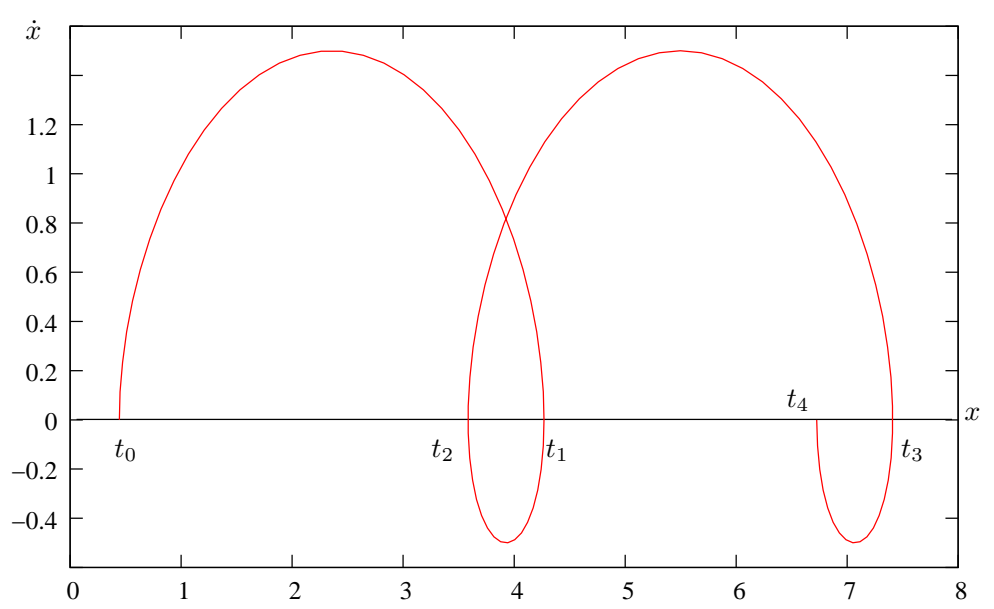

Figure 4 . Trajectory in the phase space $(x, \dot{x})$ where $x(t)>0$ for a positive cycle.

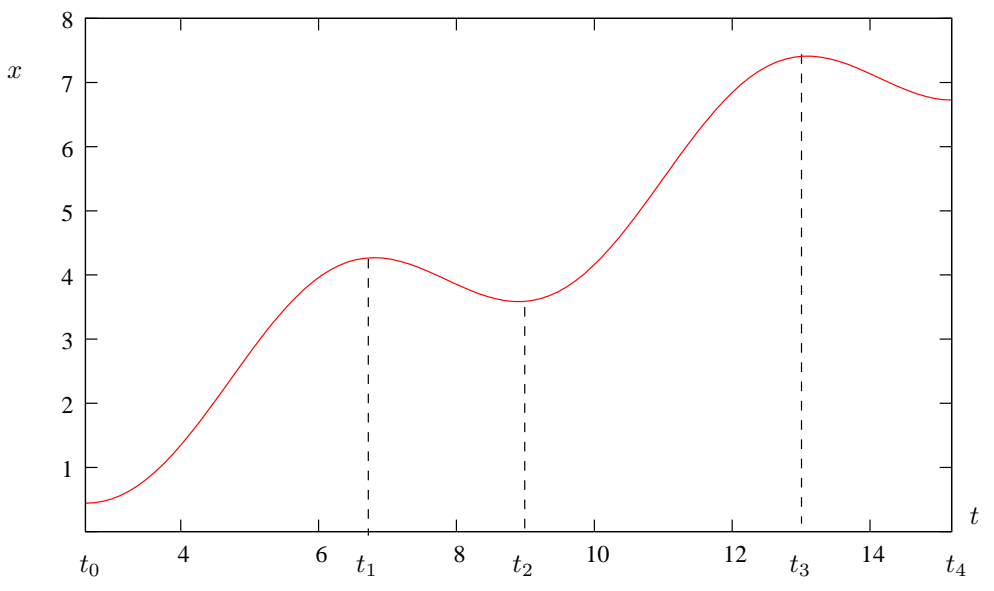

FiguRE 5. The graph of $x$ corresponding to Fig. 4.

Theorem 6.1. Under assumptions (2.1), (2.4) and the above notation, let $E_{m}$ be the energy generated by mass modulation and defined according to the energy balance (6.5) i.e.

$$
E_{m}:=E_{W}+E_{D}-E_{g} .
$$

Then the variation of $E_{m}$ during a cycle $\left(t_{0}, \ldots, t_{4}\right)$, namely

$$
\Delta E_{m}:=E_{m}\left(t_{4}\right)-E_{m}\left(t_{0}\right)
$$

satisfies

$$
\Delta E_{m}=\frac{1}{2}\left(\frac{1}{\omega}-1\right)\left(\dot{x}^{2}\left(t_{2}\right)+x^{2}\left(t_{4}\right)\right) .
$$

Proof. Let $\left(t_{0}, \ldots, t_{4}\right)$ be a positive cycle. For all $\delta t>0$,

$$
\Delta E_{g}:=E_{g}\left(t_{4}+\delta t\right)-E_{g}\left(t_{0}\right)=\int_{t_{0}}^{t_{4}+\delta t} \dot{x} g \mathrm{~d} t .
$$


By Definition 6.1, this latter integral is equal to

$$
\begin{array}{r}
\int_{\left(t_{0}, t_{1}\right) \cup\left(t_{2}, t_{3}\right) \cup\left(t_{4}, t_{4}+\delta t\right)} \dot{x}\left(\frac{1}{\omega} \ddot{x}+2 \delta \dot{x}+x\right) \mathrm{d} t+\int_{\left(t_{1}, t_{2}\right) \cup\left(t_{3}, t_{4}\right)} \dot{x}(\ddot{x}+2 \delta \dot{x}+x) \mathrm{d} t= \\
\int_{t_{0}}^{t_{4}+\delta t} 2 \delta \dot{x}^{2} \mathrm{~d} t+E_{\omega}\left(t_{1}\right)-E_{\omega}\left(t_{0}\right)+E_{\omega}\left(t_{3}\right)-E_{\omega}\left(t_{2}\right)+E_{\omega}\left(t_{4}+\delta t\right)-E_{\omega}\left(t_{4}\right) \\
+E_{1}\left(t_{2}\right)-E_{1}\left(t_{1}\right)+E_{1}\left(t_{4}\right)-E_{1}\left(t_{3}\right) .
\end{array}
$$

We have $x \dot{x}\left(t_{1}\right)=0$. If $\dot{x}\left(t_{1}\right) \neq 0$ then $x \dot{x}>0$ on $\left(t_{1}, t_{1}+\varepsilon\right)$. This contradicts (6.8) since $\left(t_{0}, \ldots, t_{4}\right)$ is a positive cycle. Thus $\dot{x}\left(t_{1}\right)=0$ and $E_{1}\left(t_{1}\right)=E_{\omega}\left(t_{1}\right)$. In a same way, $E_{1}\left(t_{3}\right)=E_{\omega}\left(t_{3}\right)$. Thus

$$
\begin{aligned}
E_{g}\left(t_{4}+\delta t\right)-E_{g}\left(t_{0}\right)=\int_{t_{0}}^{t_{4}+\delta t} 2 \delta \dot{x}^{2} \mathrm{~d} t+E_{\omega}\left(t_{4}+\delta t\right) & -E_{\omega}\left(t_{0}\right) \\
& +\left(E_{1}-E_{\omega}\right)\left(t_{2}\right)+\left(E_{1}-E_{\omega}\right)\left(t_{4}\right) .
\end{aligned}
$$

Letting $\delta \rightarrow 0^{+}$and using (6.2), the definition of $E_{W}$ and (6.5), we get (6.9). If the cycle is negative, we obtain also (6.9).

Remark 6.1. From (6.9) and the energy balance (6.5), it is clear that when $\omega<1$, mass modulation increases the energy of the system. Moreover if $x$ is periodic and the period is equal to the length of a cycle then

$$
\begin{aligned}
\Delta E_{D} & =\Delta E_{g}+\Delta E_{m} \\
& =\Delta E_{g}+\frac{1}{2}\left(\frac{1}{\omega}-1\right)\left(\dot{x}^{2}\left(t_{2}\right)+x^{2}\left(t_{0}\right)\right) .
\end{aligned}
$$

So the additional energy $\Delta E_{m}$ brought by mass modulation is transferred to the damping part of the system. This damping part is made of the damping of the power takeoff device and the hydrodynamic damping. In this sense, the new WEC proposed by Orazov et al. is more efficient than standard oscillators.

\section{REFERENCES}

[dBBCK08] M. di Bernardo, C. J. Budd, A. R. Champneys, and P. Kowalczyk. Piecewise-smooth dynamical systems, volume 163 of Applied Mathematical Sciences. Springer-Verlag London Ltd., London, 2008. Theory and applications.

[OOS10] B. Orazov, O.M. O'Reilly, and Ö. Savaş. On the dynamics of a novel ocean wave energy converter. Journal of Sound and Vibration, 329(24):5058-5069, 2010.

Laboratoire de Mathématiques et Applications

UMR 7348

Université De PoItiers \& CNRS

SP2MI - BP 30179

86962 Futuroscope Chasseneuil Cedex - France

E-mail address: Arnaud.Rougirel@math.univ-poitiers.fr 